\title{
Representation of Auditory Space by Cortical Neurons in Awake Cats
}

\author{
Brian J. Mickey and John C. Middlebrooks \\ Kresge Hearing Research Institute, University of Michigan, Ann Arbor, Michigan 48109-0506
}

We evaluated the spatial selectivity of auditory cortical neurons in awake cats. Single- and multiunit activity was recorded in primary auditory cortex as the animals performed a nonspatial auditory discrimination or sat idly. Their heads were unrestrained, and head position was tracked. Broadband sounds were delivered from locations throughout $360^{\circ}$ on the horizontal plane, and source locations were expressed in head-centered coordinates. As in anesthetized animals, the firing rates of most units were modulated by sound location, and most units responded best to sounds in the contralateral hemifield. Tuning was sharper than in anesthetized cats, in part because of suppression at nonoptimal locations. Nonetheless, spatial receptive fields typically spanned $150-180^{\circ}$. Units exhibited diverse temporal response patterns that often depended on sound location. An information-theoretic analysis showed that information transmission was reduced by $\sim 10 \%$ when the precision of spike timing was disrupted by $16-32 \mathrm{msec}$, and by $\sim 50 \%$ when all location-related variation of spike timing was removed. Spikes occurring within $60 \mathrm{msec}$ of stimulus onset transmitted the most location-related information, but later spikes also carried information. The amount of information transmitted by ensembles of units increased with the number of units, indicating some degree of mutual independence in the spatial information transmitted by various units. Spatial tuning and information transmission were changed little by an increase in sound level of $20-30 \mathrm{~dB}$. For the vast majority of units, receptive fields showed no significant change with the cat's head position or level of participation in the auditory task.

Key words: sound localization; spatial hearing; auditory cortex; unanesthetized; space perception; level invariance; spike timing

\section{Introduction}

The auditory cortex is essential for normal sound localization in mammals. Lesions of the auditory cortex in cats (Jenkins and Masterton, 1982; Jenkins and Merzenich, 1984), monkeys (Thompson and Cortez, 1983; Heffner and Heffner, 1990; Heffner, 1997), and ferrets (Kavanagh and Kelly, 1987) result in deficits in localizing sounds in the hemifield contralateral to the lesion. Numerous physiological studies using anesthetized animals are consistent with those lesion studies in that auditory cortical neurons are sensitive to the locations of sound sources, generally favoring sounds in the contralateral hemifield (for review, see Middlebrooks et al., 2001). No topographical representation of space has been demonstrated in the auditory cortex, but nearby units tend to have similar receptive fields. Some physiological results are difficult to reconcile with the ability of listeners to localize sounds accurately at virtually any location over a wide range of sound levels. Even at moderate sound levels, spatial receptive fields tend to be broad, usually spanning more than a

Received June 21, 2003; revised July 24, 2003; accepted July 27, 2003.

This work was supported by the National Institutes of Health (R01 DC00420, R01 EB00308, P41 RR09754, T32 GM07863, T32 DC0001) and by the Scottish Rite Schizophrenia Research Program. We thank Ewan Macpherson, lan Harrington, Sandy Bledsoe, Colleen Le Prell, Wayne Aldridge, Rich Hume, David Moody, and David Anderson for comments on previous versions of this manuscript; Chris Stecker, Ewan Macpherson, Shigeto Furukawa, Li Xu, and Julie Arenberg for many stimulating discussions; Zekiye Onsan for help in training animals; the Center for Neural Communication Technology and Jamie Hetke for providing multichannel probes; Chris Ellinger and Jack Briggs for help in building the experimental apparatus; and Gail Rising and Sherry Wagar for surgical expertise.

Correspondence should be addressed to John C. Middlebrooks, Kresge Hearing Research Institute, University of Michigan, Ann Arbor, Ml 48109-0506. E-mail: jmidd@umich.edu.

Copyright $\odot 2003$ Society for Neuroscience $\quad$ 0270-6474/03/238649-15\$15.00/0 hemifield of space. Moreover, spatial tuning tends to broaden and the accuracy of location coding tends to decrease at higher sound levels (Imig et al., 1990; Rajan et al., 1990; Middlebrooks et al., 1998; Reale et al., 2003; Stecker et al., 2003). A few studies using unanesthetized animals have confirmed that auditory cortical neurons are sensitive to sound location, but those studies were limited by the range of locations and sound levels tested (Eisenman, 1974; Leinonen et al., 1980; Benson et al., 1981; Ahissar et al., 1992; Recanzone et al., 2000).

Several fundamental issues remain to be addressed. First, previous studies using awake animals have tested only a limited number of frontal source locations. Given the broad spatial tuning of most units, and given the ability of animals to localize sounds at essentially any location, a fuller description of unit receptive fields is needed. Second, the dependence of spatial receptive fields on sound level has not been described in awake animals. The broadening of receptive fields with increasing sound level that is seen in anesthetized preparations conflicts with sound localization behavior, which typically maintains (or even improves) its accuracy with increasing sound level (Good and Gilkey, 1996; Macpherson and Middlebrooks, 2000; Su and Recanzone, 2001). Third, further study of topographical organization is warranted. It remains possible that the auditory cortex possesses spatial topography that is obscured by the use of anesthesia. Fourth, studies in anesthetized cats have shown that spike timing can carry as much or more location-related information as mean spike rates (Middlebrooks et al., 1994, 1998; Brugge et al., 1996; Xu et al., 1998; Furukawa and Middlebrooks, 2001, 
2002; Jenison et al., 2001; Reale et al., 2003). Temporal firing patterns are certainly different in the absence of anesthesia, yet there have been no studies of location-dependent spike patterns in awake animals. Finally, the dependence of cortical responses on behavioral state and on head position deserves more attention. Two studies have investigated these questions in awake animals. They suggest that, except for a small minority of units, spatial sensitivity is independent of the task the animal is performing (Benson et al., 1981) and that a head-centered reference frame underlies the receptive fields of auditory cortical neurons (Leinonen et al., 1980).

To address these issues, we have developed a new preparation to allow recording of unit activity from the auditory cortex of awake cats. This preparation has revealed a rich diversity of location-dependent firing patterns that have been masked previously by the use of general anesthesia.

\section{Materials and Methods}

Behavioral training. We used six female purpose-bred domestic cats (Harlan, Indianapolis, IN). All procedures were approved by the University of Michigan Committee on Use and Care of Animals. Cats had clean external ears and no obvious hearing deficits. Their ages ranged from 9 to 22 months, and their body weights ranged from 2.9 to $5.2 \mathrm{~kg}$. Five of the six cats were ovarectomized before training, with the goal of increasing day-to-day consistency of performance of the behavioral task. Cats were housed individually or in pairs in small rooms. Water was provided ad libitum. Food was withheld overnight before morning or afternoon training sessions to increase motivation. Dry cat food was provided in the home room after training; canned cat food was liquefied for use as a reinforcer during training and recording sessions.

Each cat was gradually introduced to the experimental chamber, the experimental platform, and the feeding apparatus. To reduce disturbance of the acoustic field, the experimental platform and the feeding apparatus were as small as was practical; to minimize interference with the head-tracking system (see Recording sessions), they were constructed of nonmetallic materials wherever possible. The cat sat on the experimental platform, which was a small shelf $(23 \times 44 \mathrm{~cm})$ that was covered with carpet and raised $93 \mathrm{~cm}$ above the floor of the chamber by a vertical post. The feeding apparatus consisted of a food dish placed in front of and slightly below the cat's resting head position, a spout that delivered liquid food into the dish, and a response key mounted in front of the cat's forepaws. Between reinforcements, the food dish was pneumatically lowered into a vertical cylinder, rendering the dish inaccessible to the cat; this mechanism allowed more precise temporal control of the cat's feeding behavior. A harness fit snugly around the cat's upper body and attached securely to the platform. The harness allowed the cat to sit or stand and to move its head and limbs freely, but prevented the animal from turning around or leaving the platform. The chamber was dimly lit by an incandescent bulb so that the experimental apparatus was visible. Cats were monitored continuously from outside the experimental chamber via a closed-circuit television monitor.

Each cat was trained to perform an auditory task during once- or twice-daily sessions that typically lasted 30-60 min each. The training lasted 2-10 months, depending on the animal. The cat was required to hold down the response key with a forepaw for a minimum time called the hold period. The duration of the hold period was chosen randomly on each trial (typically $8-12 \mathrm{sec}$ ). If the key was released before the end of the hold period (a false alarm), then the behavioral trial ended. Timeouts were not used. If the key was held throughout the entire hold period, then a cue stimulus, a train of clicks [rate $200 \mathrm{~Hz}$; level 20-40 dB sound pressure level (SPL)], was presented. For three cats, the cue stimulus was presented from an overhead loudspeaker; for the other three cats, it was presented from an azimuthal location chosen randomly trial to trial (elevation $0^{\circ}$ ). The duration of the cue stimulus was $0.8-2.0 \mathrm{sec}$, depending on the animal. If the key was released during the cue stimulus (a hit), a bolus of liquid food was delivered into the food dish, the cat was allowed to eat for $5 \mathrm{sec}$, and the behavioral trial ended. If the key was not released during the cue stimulus (a miss), no food was delivered, and the behavioral trial ended. The next behavioral trial began immediately if the key was still depressed or when the cat pressed the key again. Probe stimuli, which consisted of noise bursts, pure tones, and clicks, were presented both during the hold period of behavioral trials and also in between behavioral trials. The interstimulus interval was $1.5-2.0 \mathrm{sec}$; it was jittered trial to trial by $\sim 0.2 \mathrm{sec}$ to reduce the predictability of the stimulus onset.

Task performance varied widely within and between sessions. Because probe stimuli were most often $100 \mathrm{msec}$ noise bursts delivered at 20-50 $\mathrm{dB}$ SPL from loudspeakers at various locations, the behavioral task amounted to a discrimination between a $200 \mathrm{~Hz}$ click train and a broadband noise burst. The click trains resembled the interleaved noise bursts in overall distribution of spectral energy, but the longer duration and obvious tonal character of the click train made the discrimination task relatively easy. The cats tended to alternate between periods during which they were engaged in and actively performing the task and periods during which they were idle. For purposes of analysis, idle periods were defined by an absence of key-pressing activity lasting more than $\sim 20 \mathrm{sec}$. Idle periods occurred interspersed with performing periods or near the end of a session when the cat was sated. To measure task performance, we calculated the hit rate (hits per cue stimulus), the false-alarm rate (false alarms per probe stimulus), the miss rate (misses per cue stimulus), and the overall percentage correct (per cue or probe stimulus). During performing periods, the median (and range) for hit, miss, and false-alarm rates was $85 \%(47-100 \%), 15 \%(0-51 \%)$, and $1.4 \%(0-16 \%)$, respectively, and the overall percentage correct was $97 \%$ (79-99\%).

Surgery. After the cats were trained in the behavioral task, a fixture was implanted on the skull. The skull fixture was cast in aluminum from a plastic model that fit the curvatures of a cat's skull. The casting was then machined and anodized. The fixture, which ultimately weighed $\sim 20 \mathrm{gm}$, was composed of a cylindrical recording chamber (diameter $\sim 15 \mathrm{~mm}$, depth $\sim 1 \mathrm{~cm}$ ) to be situated over the auditory cortex and an adjacent slot designed to hold the external connector for the recording probe (see Probe placement).

The skull fixture was implanted under aseptic conditions in an approved surgical suite. Antibiotics (cephazolin, $35 \mathrm{mg} / \mathrm{kg}$ body weight, i.m. or s.c., every $12 \mathrm{hr}$ ) were administered prophylactically for $3 \mathrm{~d}$ starting the day before surgery. Anesthesia was induced either with injected ketamine $(10 \mathrm{mg} / \mathrm{kg})$ and xylazine $(1 \mathrm{mg} / \mathrm{kg})$ or with isoflurane $(4-5 \%$, with $33 \% \mathrm{O}_{2}$ and $67 \% \mathrm{~N}_{2}$. The airway was intubated and isoflurane anesthesia was maintained throughout the procedure. The scalp was incised at the midline, and portions of the scalp and underlying temporalis muscle were removed from the right side. An opening $\sim 1 \mathrm{~cm}$ in diameter was created in the skull using a dental bur and Rongeurs, and the dura that covered the right middle ectosylvian gyrus was exposed. The skull fixture was attached over the skull opening with stainless steel screws and dental acrylic. Gentamicin or tobramycin $(\sim 0.1 \mathrm{mg}$, ophthalmic preparation) was applied to the exposed dura for antibiotic prophylaxis, and the recording chamber was filled with SILASTIC, a silicone elastomer (World Precision Instruments, Sarasota, FL). The scalp was sutured closed around the skull fixture, and cyanoacrylate glue was applied between the wound margin and skull fixture. A protective lid covered the skull fixture and its contents. The wound was allowed to heal for 2-4 weeks before placement of a recording probe. The pinnae were well preserved despite the surgery. In two of the six animals, the resting orientation of the right pinna was mildly deviated laterally; in no animal was the mobility of the pinna altered appreciably.

Probe placement. Probe placement was performed in the laboratory under general anesthesia. Anesthesia was induced with a subcutaneous or intramuscular injection of ketamine $(10 \mathrm{mg} / \mathrm{kg})$ and xylazine $(1 \mathrm{mg} / \mathrm{kg})$. At the beginning of the procedure, atropine $(0.1 \mathrm{mg})$ and cephazolin $(35$ $\mathrm{mg} / \mathrm{kg}$ ) were administered intramuscularly. Often a supplemental halfdose of ketamine-xylazine was given 45-60 min later. The plug of SILASTIC was removed, granulation tissue was cleared, and a small opening $(1-5 \mathrm{~mm})$ was created in the dura. An external connector was installed in the skull fixture, and the attached multichannel probe was manually inserted through the dural opening and into active cortical layers. Gentamicin or tobramycin $(\sim 0.1 \mathrm{mg})$ was applied topically to the 
A

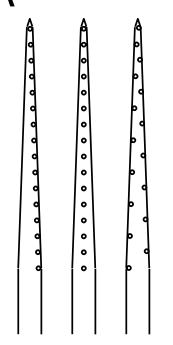

$\mathrm{B}$

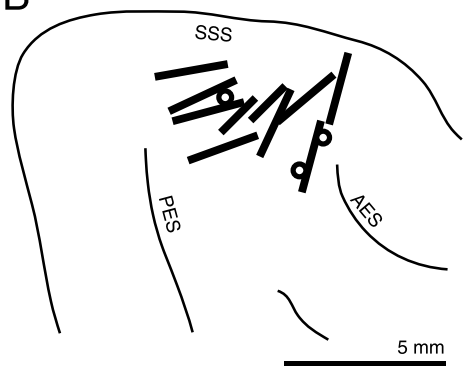

Figure 1. Placement of multichannel recording probes in the auditory cortex. A, Schematic diagram showing the three site geometries used. Probes were $5 \mathrm{~mm}$ long, $15 \mu \mathrm{m}$ thick, and 200 $\mu \mathrm{m}$ wide, with a taper down to $33 \mu \mathrm{m}$. The longitudinal separation of adjacent recording sites (small circles) was 150 or $100 \mu \mathrm{m}$. B, Thirteen probe placements. The positions of multichannel electrodes are shown in relation to major sulci: the superior sylvian sulcus (SSS), the posterior ectosylvian sulcus (PES), and the anterior ectosylvian sulcus (AES). Because sulcal patterns varied between animals, probe positions are only approximate. Ten placements were tangential to the cortical surface (black bars) and two were radial or oblique (circles). Scale bar, $5 \mathrm{~mm}$.

brain. Finally, the recording chamber was refilled with SILASTIC, which, after hardening, fixed the probe in place, sealed the opening, and prevented the brain from herniating. At the end of the procedure, which lasted $1-2 \mathrm{hr}$, yohimbine $(0.4 \mathrm{mg} / \mathrm{kg})$ was usually injected intramuscularly to reverse the effects of xylazine and thereby speed recovery. After recording daily for a period of several days to several weeks (see Recording sessions), the probe and connector were removed and a new probe and connector were installed.

Probes were silicon-substrate 16-channel electrodes (Anderson et al., 1989) designed for chronic extracellular recording and provided by the University of Michigan Center for Neural Communication Technology. Each probe consisted of a single shank with 16 recording sites (impedances $1-5 \mathrm{M} \Omega$ at $1 \mathrm{kHz}$ ). We used probes with longitudinal site separations of 100 or $150 \mu \mathrm{m}$, but with three slightly different geometries (Fig. $1 \mathrm{~A}$ ). Probes were placed in the middle ectosylvian gyrus usually tangentially (at an angle of $10-30^{\circ}$ with respect to the cortical surface) but in a few cases at a steeper angle $\left(>45^{\circ}\right)$. The number of successful probe placements per animal ranged from 1 to 3 , totaling 13 across all six animals (Fig. $1 B$ ). The number of sites with responsive units ranged from 1 to 16 per probe placement (median, 8.5).

Stimulus generation. Stimuli were presented under free-field conditions with an apparatus that has been described previously (Middlebrooks et al., 1998). A sound-attenuating chamber (inner dimensions $2.6 \times 2.6 \times 2.5 \mathrm{~m}$ ) was lined with sound-absorbing foam to suppress reflections. The ambient sound level in the chamber was $20 \mathrm{~dB}$ SPL (A-weighted). Two-way coaxial loudspeakers (Pioneer TS-879 or similar) were located $1.2 \mathrm{~m}$ from the cat's head on a horizontal circular hoop. The location directly ahead of the animal was designated as $0^{\circ}$ azimuth, $0^{\circ}$ elevation. The hoop held loudspeakers at $0^{\circ}$ elevation at various azimuths $\left(-160^{\circ}\right.$ to $+180^{\circ}$ in $20^{\circ}$ steps); azimuths to the right were assigned positive values. Experiments were controlled by custom Matlab software (The Mathworks, Natick, MA) running on a Pentium-based personal computer with signal processing instruments from Tucker-Davis Technologies (Gainesville, FL).

Physiological experiments used noise bursts, pure-tone bursts, clicks, and click trains. The stimulus passband was $0.5-30 \mathrm{kHz}$ or, in later experiments, $0.2-30 \mathrm{kHz}$. Because the transfer functions generally differed between loudspeakers, each loudspeaker was calibrated individually by obtaining an impulse response (Zhou et al., 1992). Stimuli were created by convolution of the intended signal with the inverse impulse response of the intended loudspeaker. Noise bursts were Gaussian distributed with abrupt onsets and offsets; a different random sample was presented on each trial. Pure-tone bursts were ramped on and off with $5 \mathrm{msec}$ raisedcosine functions. Click trains consisted of repeated $10 \mu$ sec impulses. Stimulus waveforms were generated with 16-bit precision at a sampling rate of $100 \mathrm{kHz}$.

Recording sessions. The same behavioral conditions existed during re-

cording sessions as during training sessions (see Behavioral training), except that during the recording sessions a headstage and a head-tracker receiver were mounted on the skull fixture to allow physiological recordings and measurements of head position and orientation. The headstage was mechanically protected and electrically shielded by an aluminum housing (dimensions $0.8 \times 2.5 \times 5.5 \mathrm{~cm}$ ) that was attached to the skull fixture during recording sessions. The head-tracker receiver (dimensions $1.5 \times 2.0 \times 2.0 \mathrm{~cm}$ ) was part of an electromagnetic tracking system (Polhemus Fastrak, Colchester, VT) used to measure the position and orientation of the cat's head. The receiver was attached via nylon spacers to the headstage housing; the spacers minimized interference between the headstage and head-tracker. Flexible cables from the headstage and head-tracker led unobtrusively down the cat's back. During a typical session, the cat spent some time engaged in the auditory task and the rest of the time idle. To maximize the amount of physiological data collected, unit activity was recorded both while the cat performed the task and while the cat was idle. Frequent movements of the head and body indicated that the cats were awake during idle periods.

During initial characterization of units, thresholds were estimated by presenting broadband noise bursts (100 msec duration) from overhead or frontal locations at various sound levels. Then, noise bursts were delivered every $1.5-2.0 \mathrm{sec}$ from 1 of 18 loudspeakers distributed over a $360^{\circ}$ range of azimuth. Sounds were presented at a single level $10-40 \mathrm{~dB}$ above unit threshold or at three or four levels in $10 \mathrm{~dB}$ steps. Thresholds sometimes varied among units within a penetration; in those cases, an average threshold was used to select stimulus levels. Stimuli were presented in random order such that all levels and locations were tested once before all stimuli were repeated again in a different random order. The total number of stimulus presentations at each sound level ranged from 160 to 596 across recording sessions. Stimulus locations were grouped into 12 bins (see Data analysis), so the number of repetitions of each combination of sound level and location was 13-50 (averaged across locations). Activity was recorded at 16 probe sites simultaneously. For $100 \mathrm{msec}$ stimuli, we recorded during the $200 \mathrm{msec}$ period beginning at stimulus onset or the $250 \mathrm{msec}$ period beginning $50 \mathrm{msec}$ before stimulus onset. Spike times were expressed relative to the onset of digital-to-analog conversion; therefore, latencies include $3.5 \mathrm{msec}$ of acoustic travel time. The activity at each site was amplified, high-pass filtered above $200 \mathrm{~Hz}$ to remove slow potentials, and digitized with 16-bit precision at a sampling rate of 25 $\mathrm{kHz}$. The signal was then sharply low-pass filtered below $6 \mathrm{kHz}$ to prevent aliasing, resampled at $12.5 \mathrm{kHz}$, and stored on the computer hard disk for later analysis.

The position and orientation of the cat's head were measured during recording sessions. On each trial, head coordinates were acquired the onset and offset of the physiological recording. Head orientation was expressed in terms of azimuth, elevation, and roll angles. Figure $2 \mathrm{~A}$ shows the distributions of head orientation angles across all recording sessions in all animals. Trials on which the head assumed an extreme orientation (elevation less than $-80^{\circ}$ or more than $80^{\circ}$, or roll less than $-80^{\circ}$ or more than $80^{\circ}$ ) were removed before analysis. Ninety percent of azimuth, elevation, and roll measurements fell within a range of $173^{\circ}$, $55^{\circ}$, and $65^{\circ}$, respectively. Calibration procedures indicated that the head-tracking system suffered from systematic errors in azimuth and elevation, probably because of metallic objects (such as the skull fixture) that could not be eliminated from the environment. For orientations in the frontal hemifield, the error in azimuth was as high as $6^{\circ}$ for extreme lateral azimuths, and the error in elevation was as high as $12^{\circ}$. Because this study is concerned with an angular resolution two- to threefold greater than these errors, we have not attempted to correct for them.

We were concerned that the neuronal responses that we measured might be influenced by active movements of the pinnae (Populin and Yin, 1998), either during a stimulus presentation or between stimuli. Although movements of the cat's pinnae were not routinely measured in the current study, casual observations suggested that movements were slight and infrequent. Closer study of video recordings of one cat showed no apparent movements of the pinnae relative to the head except for occasional orientating toward the click-train cue. The infrequency of pinna movements may be related to the nonspatial behavioral task in which the cats were engaged. Head movements during stimulus presen- 
tations were similarly negligible, as evidenced by video recordings and by comparison of head-tracker measurements at the onset and offset of physiological recordings.

Spike sorting and unit characterization. Spike sorting was performed off-line using custom software based on principal component analysis of spike shape using a procedure similar to one described previously (Furukawa et al., 2000). Multichannel waveforms were first subjected to a de-noising procedure that removed activity that was correlated across sites (Bierer and Anderson, 1999). De-noising effectively eliminated spikes recorded on neighboring sites, so multiunit recordings from sites near each other did not share spikes in common. Candidate spikes were identified in the waveforms using a criterion based on peak amplitudes. Principal component analysis was applied to candidate spikes, and classes of spikes were manually selected from scatterplots of the first two or three principal-component weights. The quality of unit isolation was characterized on the basis of those scatterplots and on histograms of interspike intervals. Single units (SUs) were identified by discrete clustering in principal component space and a lack of interspike intervals $<1 \mathrm{msec}$ (Fig. $3 A, C$ ). More often we recorded multiunit clusters (MUs). In those cases, either spikes could not be ascribed reliably to a single neuron or they clearly originated from two or more neurons (Fig. $3 B, D$ ). In the present study, both SUs and MUs are referred to as "units." Among the sample of 125 units analyzed here, 28 were SUs and 97 were MUs. The activity across electrode sites typically varied day to day, suggesting that the probe was moving relative to the brain or that the local environment was changing as a result of probe implantation. Occasionally we recorded on consecutive days what appeared to be the same $\mathrm{SU}$; because this was impossible to verify, however, we used data from only a single selected recording session (30-60 min long) for each unit. Spike shapes and spike count statistics tended to be stable over this period, with the exception of one penetration, which was eliminated from further analysis.

Electrodes were positioned in active cortical layers of the middle ectosylvian gyrus (Fig. $1 \mathrm{~B}$ ). Units tended to respond most strongly to high frequencies. In response to pure tone bursts (frequency $1-30 \mathrm{kHz}$, at various sound levels, usually presented from an overhead loudspeaker), units typically fired a burst of spikes after the onset of the sound. We measured frequency response areas for 90 units from eight probe placements in five animals. Of the 90 units, 11 did not respond to pure tones and 6 exhibited complex response areas. Best frequencies of the remaining 73 units ranged from 2.5 to $30 \mathrm{kHz} ; 90 \%$ of units had best frequencies of $\geq 7.5$ $\mathrm{kHz}$. Extensive mapping of best frequencies was not possible given the limited number of cortical sites examined in each animal. Therefore, we were not able to determine with certainty the cortical field of each recorded unit. Nonetheless, a comparison of the positions of our electrodes with published maps of cortical fields in anesthetized cats (Reale and Imig, 1980) suggests that most of our unit population was in area A1. Our sample likely also included some units from the second auditory area (A2) and the anterior auditory field (AAF).

Data analysis. In the absence of evidence to the contrary, we presumed that neurons represented sound locations in head-centered coordinates. Therefore, on each trial, we calculated the stimulus location relative to the orientation of the cat's head. This transformation was achieved by expressing the three-dimensional position of the loudspeaker in terms of the six-dimensional reference frame of the cat's head (azimuth, elevation, roll, $\mathrm{x}$-position, y-position, and z-position). The two-dimensional direction of the loudspeaker was then expressed in terms of horizontal polar coordinates, i.e., lateral angle $(\theta)$ and polar angle $(\varphi)$. The range of $\theta$ was $-90^{\circ}$ to $+90^{\circ}$; negative values indicated locations in the left hemisphere, positive values, the right hemisphere. The range of $\varphi$ was $-90^{\circ}$ to $+270^{\circ}$; $-90^{\circ}$ (and $+270^{\circ}$ ) was downward, $0^{\circ}$ toward the front, $+90^{\circ}$ upward, and $+180^{\circ}$ to the rear. (The angles $\theta$ and $\varphi$ are equivalent to latitude and longitude, respectively, on a globe, where the interaural axis corresponds to the globe's axis of rotation.) This coordinate system is appealing for studies of spatial hearing because interaural time and level differences vary mainly with $\theta$, whereas monaural spectral cues vary mainly with $\varphi$ (Morimoto and Hitoshi, 1984; Middlebrooks, 1992). In the current study, the head-centered locations of sound sources fell near, but not exactly on, the horizontal plane throughout $360^{\circ}$. We therefore sought a single angular measure that ranged from $-180^{\circ}$ to $+180^{\circ}$. Azimuth would seem a natural choice, but it has the undesirable feature that sources with the same azimuth but different elevations generally produce different interaural cues. Therefore, for the purposes of our study, we 

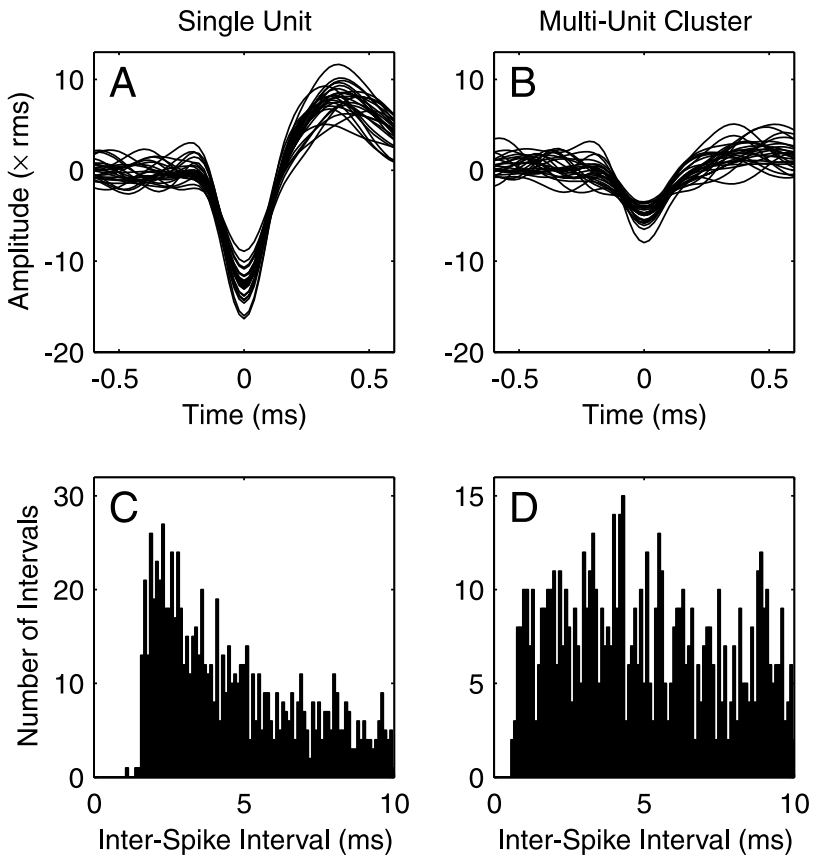

Figure 3. Unit isolation by analysis of waveforms and interspike interval histograms. $A$, Spike waveforms of a SU. Twenty spikes were chosen randomly from a single recording session. Signal amplitude is expressed relative to the root mean square level of the background noise. $B$, Spike waveforms of a MU. MUs typically had indistinct spike shapes and low signal-to-noise ratios. C, First-order distribution of interspike intervals for the SU shown in $A$. SUs lacked interspike intervals under $1 \mathrm{msec}$. $D$, Interspike interval histogram for the MU shown in $B$.

defined the "location" of the stimulus $(\alpha)$ as follows: front hemisphere, $\alpha=$ $\theta$; right rear quandrant, $\alpha=180^{\circ}-\theta$; left rear quandrant, $\alpha=-180^{\circ}-\theta$.

Note that $\alpha$ collapses across polar angle except to preserve the distinction between front and back. For sources on the horizontal plane $\left(\varphi=0^{\circ}\right.$ or $\left.180^{\circ}\right), \alpha$ is equivalent to azimuth. Source locations were finally quantized into $1230^{\circ}$-wide bins. This bin size was chosen because for some units smaller bin sizes resulted in too few trials per bin. Figure 2, $B$ and $C$, illustrates the effect of transforming from room-centered to headcentered stimulus locations.

Mean evoked spike rates were calculated by finding the time-averaged spike rate for the period from 10 to $160 \mathrm{msec}$ after stimulus onset and averaging across trials for each stimulus condition. The spontaneous rate of each unit was calculated by averaging across all trials the spike rate during the $50 \mathrm{msec}$ period immediately preceding stimulus onset or, for some units, during the $10 \mathrm{msec}$ period immediately after stimulus onset. Evoked responses were never seen in the first $10 \mathrm{msec}$, which included $\sim 3.5 \mathrm{msec}$ of acoustic travel time.

We characterized the spatial receptive field of each unit using three measures: modulation depth, tuning width, and best location. These statistics were used in a previous study from our laboratory that used anesthetized cats (Middlebrooks et al., 1998) but are generalized here to accommodate non-zero spontaneous rates. A rate versus location profile $r(\alpha)$ was computed for each unit (see Fig. $6 A$ for an example). The modulation depth was defined as $\left(r_{\max }-r_{\min }\right) / k$, where $r_{\min }$ is the minimum rate and $r_{\max }$ is the maximum rate. The factor $k$ is equal to $r_{\max }-$ $r_{\mathrm{s}}$ or $r_{\min }-r_{\mathrm{s}}$, whichever is greater, where $r_{\mathrm{s}}$ is the spontaneous rate. The modulation depth could range from 0 to $200 \%$. Note that when the spontaneous rate equaled zero, the maximum possible modulation depth was $100 \%$. A modulation depth of $200 \%$ resulted when the maximum rate was as far above the spontaneous rate as the minimum rate was below it. This measure can be sensitive to noise, so we conservatively modified the calculation to incorporate $\sigma(\alpha)$, the SEM of $r(\alpha)$ : rather than simply using $r_{\min }$, we used the minimum of $r(\alpha)+\sigma(\alpha)$; and rather than $r_{\max }$, we used the maximum of $r(\alpha)-\sigma(\alpha)$. The tuning width was defined as $360^{\circ} \times f$, where $f$ is the fraction of locations at which $r(\alpha)$ was above the half-maximal rate, $\left(r_{\max }-r_{\mathrm{s}}\right) / 2$. The tuning width was undefined in cases where $r_{\max }<r_{\mathrm{s}}$. The best location was calculated when the modulation depth was $>50 \%$. First, we defined a peak as the set of responses at one or more contiguous locations near $r_{\max }$ that exceeded a criterion spike rate, $r_{\min }+3\left(r_{\max }-r_{\min }\right) / 4$. Then, we computed the spike rateweighted vector sum of these responses, plus the two subcriterion responses on either side of the peak. In the vector sum, $r$ was used as the magnitude of the vector and $\alpha$ was used as the direction. The direction of the resultant vector was taken as the best location. Thus the best location was a sort of spike rate-weighted centroid.

To estimate the amount of location-related information transmitted by unit responses, the spike patterns of each unit were analyzed in three steps: (1) we divided trials into two sets of equal size and computed average response patterns for each set; (2) we used a pattern-recognition algorithm to test the consistency of stimulus-specific responses; (3) we computed the amount of stimulus-related information transmitted by the pattern recognition.

In the first step, we randomly divided the set of trial-by-trial responses into two sets of equal size, $\mathrm{A}$ and $\mathrm{B}$, and then averaged across trials for each stimulus location $\alpha$ to obtain mean response measures $R_{\mathrm{A}}(\alpha)$ and $R_{\mathrm{B}}(\alpha)$. The response measure consisted of a multidimensional spike density function (versus poststimulus time) or a unidimensional measure (e.g., spike count). Several different multidimensional response measures were used, as described in a previous study (Furukawa and Middlebrooks, 2002). Full spike patterns consisted of spike density functions over the range of time 10-200 msec after stimulus onset. Spike density functions were calculated by representing the spike pattern on each trial as a series of zeros and ones (resolution $0.1 \mathrm{msec}$ ), convolving with a Gaussian function (SD $1 \mathrm{msec}$ ), resampling at $500 \mathrm{~Hz}$, and finally averaging across trials. Windowed spike patterns were spike density functions over a $40 \mathrm{msec}$ window centered at various peri-stimulus times. Shuffled spike patterns resembled full spike patterns except that the times of spikes were shuffled randomly among trials to remove stimulus-related differences in spike timing. The randomization was accomplished by compiling a distribution of spike times across all stimulus locations and then replacing, trial by trial, each spike with a spike drawn randomly without replacement from that distribution of spike times. Interval-shuffled spike patterns systematically degraded the precision of spike times. The poststimulus time range of $10-200 \mathrm{msec}$ was first divided into equal time intervals of $1,2,4,8,16,32,64$, or $128 \mathrm{msec}$. Spike times were then shuffled randomly within each interval as in the shuffled spike pattern manipulation. First-spike patterns were formed by eliminating all but the first spike recorded on each trial and then calculating spike density functions over the time range of $10-200 \mathrm{msec}$. To form ensemble spike patterns, the full spike patterns of two or more units were concatenated end to end. In addition, we calculated several unidimensional response measures. The spike count was the number of spikes per trial recorded over the time range of $10-200 \mathrm{msec}$, averaged across trials. The first-spike latency was the geometric mean (across trials) of the latency of the first spike within the range of $10-200 \mathrm{msec}$. The spike dispersion was calculated by pooling across trials all spike times within the range of 10-200 msec and then computing the SD.

In the second step of the analysis, we performed pattern recognition on mean response measures $R_{\mathrm{A}}$ and $R_{\mathrm{B}}$ using a probabilistic neural network constructed using the Matlab Neural Network Toolbox (Demuth and Beale, 2000). Previous studies from our laboratory used different types of networks (Middlebrooks et al., 1994, 1998; Xu et al., 1998; Furukawa et al., 2000; Mickey and Middlebrooks, 2001; Furukawa and Middlebrooks, 2002; Stecker et al., 2003). In the current study, a probabilistic neural network was favored because it is relatively simple conceptually, it does not require training, it has only one arbitrary parameter (the spread factor), and it produces quantized outputs amenable to informationtheoretic analysis. The input to the network was a multidimensional or unidimensional mean response measure (described above). The first layer consisted of a radial basis layer of 12 units, one for each source location. The second layer, a competitive layer of 12 units, produced an output that corresponded to 1 of the 12 source locations. The spread factor was chosen empirically to maximize the accuracy of pattern recognition; a change in the spread factor of two orders of magnitude was 

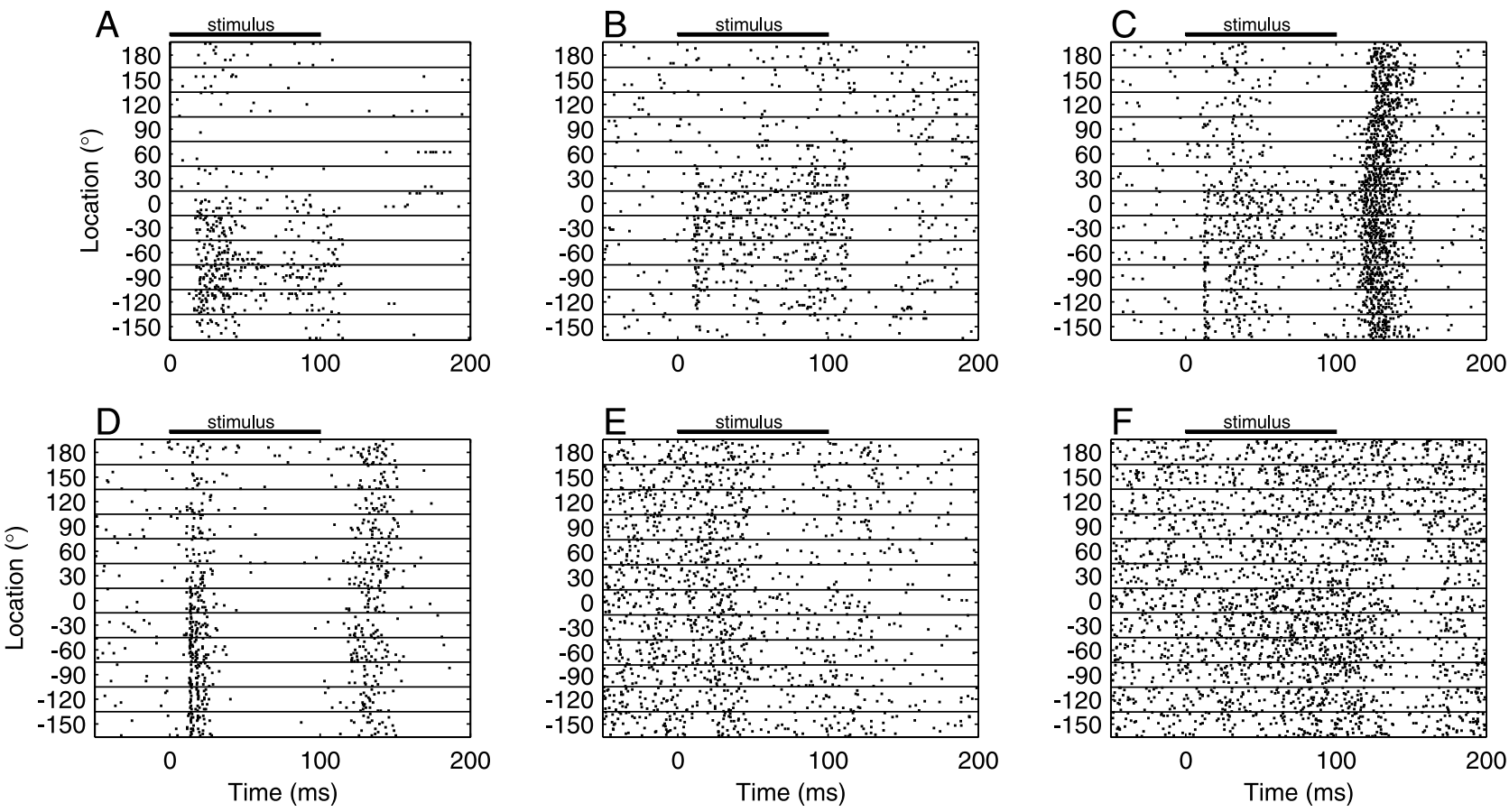

Figure 4. Diversity of temporal responses among cortical units. Each panel illustrates the responses of one unit. Each row of dots represents a train of spikes recorded on a single trial as a function of peristimulus time. Responses are ordered by sound-source location. Note that the stimulus locations lay on a circle but are unwrapped in this representation. Black bars show the time of stimulus presentation. Ten or 20 trials are shown for each location. A, SU with onset responses to contralateral sounds. B, SU with sustained responses to frontal contralateral sounds. C, MU with strong offset responses and weaker onset responses. D, MU with strong responses to both onset and offset, and a contralateral preference. $E$, MU with a suppressive onset response and no spatial sensitivity. $F$, MU with a suppressive offset response.

needed to appreciably reduce the accuracy. The weights and biases of the radial basis layer were calculated from the mean response $R_{\mathrm{A}}$ and the corresponding source locations; after the weights and biases were assigned, input of $R_{\mathrm{A}}$ resulted in outputs $Y_{\mathrm{AA}}$ that corresponded to the true source locations. To characterize how consistent $R_{\mathrm{B}}$ was with $R_{\mathrm{A}}$, the mean response $R_{\mathrm{B}}$ was presented to the network and the output $Y_{\mathrm{AB}}$ was recorded. $R_{\mathrm{A}}$ and $R_{\mathrm{B}}$ were then interchanged and an output $Y_{\mathrm{BA}}$ was obtained in a similar manner. The more closely that $R_{\mathrm{A}}$ and $R_{\mathrm{B}}$ resembled one another, the more closely $Y_{\mathrm{AB}}$ and $Y_{\mathrm{BA}}$ estimated the true stimulus locations. To reduce noise, the entire procedure was repeated a total of 50 times (i.e., 50 different randomly derived mean responses, $R_{\mathrm{A}}$ and $R_{\mathrm{B}}$, were analyzed), yielding 100 outputs for each location.

In the third step of the analysis, we estimated, for each unit and each response measure, the average amount of information transmitted about stimulus location. First, a $12 \times 12$ confusion matrix was constructed from the 100 network estimates for each location. Element $i, j$ of the confusion matrix consisted of the number of network outputs at location $i$ for the true stimulus location $j$. The accuracy of network estimates was characterized by computing the mutual information (Rieke et al., 1997), i.e., the transmitted information:

$$
T=\sum_{i} \sum_{j} p_{i j} \log _{2}\left(p_{i j} /\left(p_{i} p_{j}\right)\right) .
$$

In this expression, $p_{i}$ is the marginal probability of network output $i, p_{j}$ is the marginal probability of stimulus $j$, and $p_{i j}$ is the joint probability of network output $i$ and stimulus $j$. The more closely that network outputs estimated the true stimulus locations, the greater was $T$. In general, $T$ was greatest when average unit responses for various stimulus locations were easily discriminable; $T$ was near zero when responses varied little between stimulus locations. This method of calculating transmitted information overestimates the information in the case of random input data. For that reason we ultimately used a corrected transmitted information $\hat{T}=T-$ $T_{0}$, where $T_{0}$ was determined by a control analysis. In that analysis, the trial-by-trial association of stimulus location with unit responses was reassigned randomly. The subsequent three-step analysis was identical to that used to determine $T$. The median value of $T_{0}$ across all units was 0.4 bits for the full spike pattern condition. Given 12 stimulus locations, $\hat{T}$ theoretically ranges from 0 to 3.58 bits [i.e., $\left.\log _{2}(12)\right]$. We consider the estimate $\hat{T}$ to be a lower bound on the true amount of transmitted information, because the architecture and training of the artificial neural network were likely suboptimal in many cases.

\section{Results}

We recorded unit activity from the auditory cortex of awake cats. Brief broadband noise bursts (100 msec in duration) were presented from loudspeakers at various locations while unit activity was recorded at $1-16$ cortical sites. Roughly $20 \%$ of recording sites with spike activity did not show evoked responses to broadband noise; those units are not considered further. The data set described here consists of 125 units, 28 SUs and 97 MUs, that responded to broadband noise bursts. Most of our unit population was from area A1, but our sample may also have included some units from areas A2 and AAF. Among the population, the range of spontaneous rates was $1.8-30 \mathrm{sec}^{-1}$ (median, $8.9 \mathrm{sec}^{-1}$ ) for SUs and $0.37-35 \mathrm{sec}^{-1}$ (median, $10 \mathrm{sec}^{-1}$ ) for MUs.

In response to $100 \mathrm{msec}$ noise bursts, units showed a wide variety of spatial and temporal response patterns. We encountered units that responded primarily to the onset of the stimulus (Fig. 4A), units that responded in a sustained manner throughout the duration of the stimulus (Fig. $4 B$ ), and units that responded most strongly to the offset of the stimulus (Fig. 4C), as well as units that responded strongly to both the onset and offset of the stimulus (Fig. 4D). In addition we found units with activity that was suppressed below the spontaneous rate after stimulus onset (Fig. 4E) or offset (Fig. $4 F$ ). This diversity of temporal responses was seen even among adjacent units recorded simulta- 

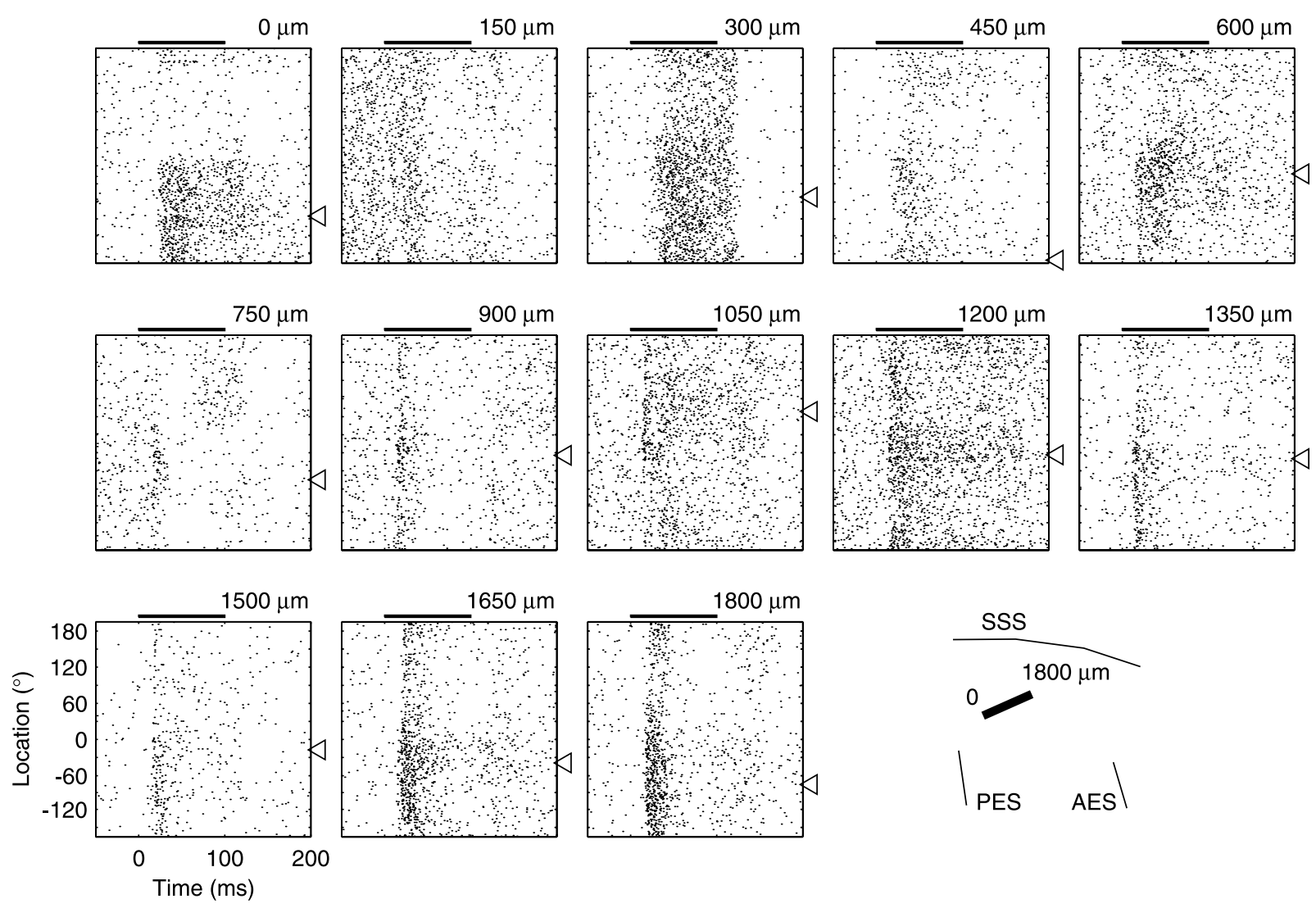

Figure 5. Responses of 13 simultaneously recorded units. Each panel shows the responses of a multiunit cluster as a function of peristimulus time and sound-source location (scales in the bottom left panel). The conventions of Figure 4 are used, except that horizontal lines between stimulus locations are omitted for clarity. These units were recorded simultaneously on a multichannel probe placed in the auditory cortex, as illustrated by the black bar in the bottom right panel. Recording sites were $150 \mu \mathrm{m}$ apart and spanned a distance of $1800 \mu \mathrm{m}$. The panels are arranged according to cortical place, which is noted at the top right of each panel, from ventrocaudal to dorsorostral sites. Open triangles indicate the best location of each unit. AES, Anterior ectosylvian sulcus; PES, posterior ectosylvian sulcus; SSS, superior sylvian sulcus.

neously on a single recording probe (Fig. 5). In many cases, the temporal pattern of the response depended on the stimulus location, suggesting that the timing of spikes carried location-related information.

\section{Spatial receptive fields}

To characterize our unit population, we first disregarded spike timing and considered only mean firing rates. Maximal evoked firing rates ranged 5.5-90 $\mathrm{sec}^{-1}$ (median, $17 \mathrm{sec}^{-1}$ ) for SUs and $3.2-83 \mathrm{sec}^{-1}$ (median, $23 \mathrm{sec}^{-1}$ ) for MUs. The spatial receptive field of a representative neuron is shown in Figure 6A. Many units resembled this example in that they were clearly selective in their responses, firing at high rates for a restricted range of source locations. Most such selective units appeared to prefer contralateral or frontal locations. Although many units had a preferred source location, practically all units responded appreciably to other, usually nearby, locations as well. That is, receptive fields were broad, typically encompassing a quadrant or hemifield of space. A minority of units exhibited little or no variation of firing rate as a function of source location.

The spatial receptive field of each unit was characterized by calculating three statistics: modulation depth, tuning width, and best location (defined in Materials and Methods). The modulation depth indicated the magnitude by which mean firing rate varied across source locations. A modulation depth of $0 \%$ indicated no variation; $100 \%$ indicated a response above the spontaneous rate at some locations and a response at the spontaneous rate at others; $200 \%$ indicated response enhancement above the spontaneous rate at some locations and response suppression of equal magnitude at other locations. Among our unit population, the modulation depth ranged from 17 to $195 \%$ (Fig. $6 B$ ). The median value was $94 \%$ for SUs and $77 \%$ for MUs. Only five SUs (18\%) and 22 MUs (23\%) had modulation depths < $<0 \%$, indicating little variation of firing rate with location. Thirty-nine percent of SUs and $30 \%$ of MUs had modulation depths $>100 \%$, indicating suppression at some locations. The breadth of spatial tuning was quantified by calculating a tuning width for each unit. A hypothetical unit that responded above its half-maximal rate for only one location would have a tuning width of $30^{\circ}$, whereas a unit that responded above its half-maximal rate to all locations would have a tuning width of $360^{\circ}$. Among 119 units, tuning widths fell across the entire range, from $30^{\circ}$ to $360^{\circ}$. The median was $150^{\circ}$ for SUs and $180^{\circ}$ for MUs (Fig. 6C). Narrow tuning was uncommon: only $30 \%$ of SUs and $13 \%$ of MUs had a tuning width of $\leq 60^{\circ}$. To estimate the preferred stimulus location of each unit, we computed the best location, an average location weighted by spike rate. For 27 units $(22 \%)$, the firing rate was not appreciably modulated by location, so a best location could not be computed. Among the remaining 98 units, best locations were distributed widely (Fig. 6D) but most fell within the hemifield contralateral to the recording site ( $83 \%$ of SUs; $75 \%$ of MUs). The quadrant that contained the greatest percentage of best locations was centered at $-48^{\circ}$ for SUs ( $57 \%$ of units) and $-18^{\circ}$ for MUs (53\% of units). 

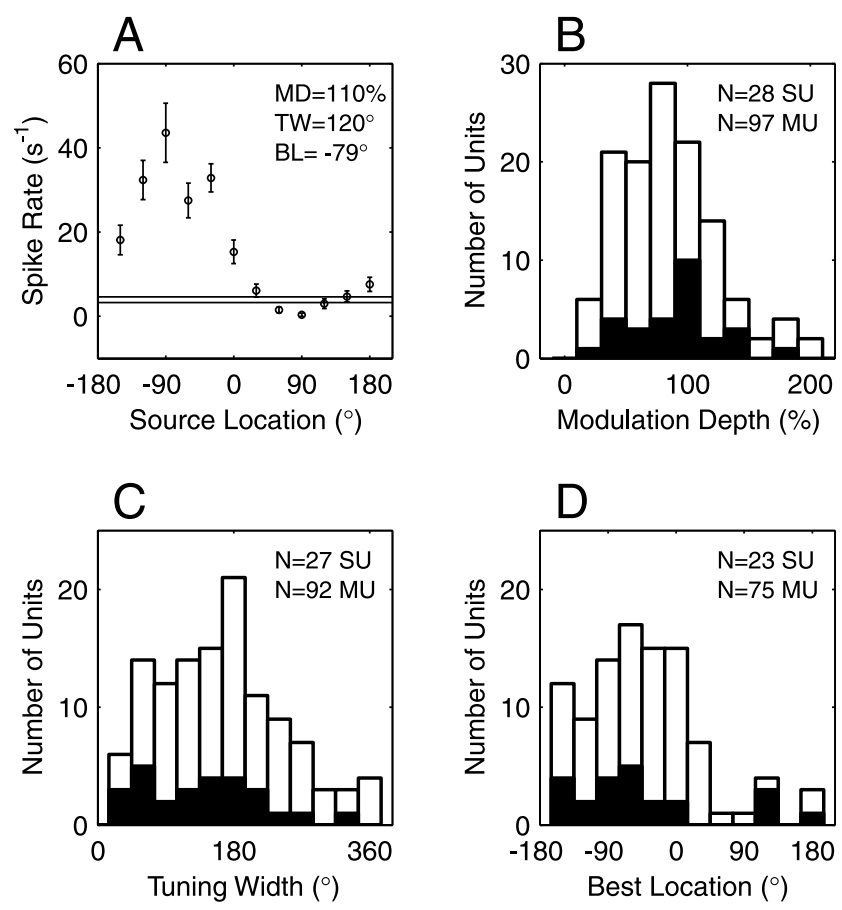

Figure 6. Analysis of spatial receptive fields. A, Spatial tuning curve of one SU. The mean firing rate (circles) and SEM (error bars) are plotted as a function of source location. Responses were averaged over the time period $10-160 \mathrm{msec}$ after stimulus onset. The thin horizontal lines indicate the spontaneous rate \pm 1 SE. This unit had a modulation depth (MD) of $109 \%$, a tuning width (TW) of $120^{\circ}$, and a best location (BL) of $-79^{\circ}$. B, Distribution of modulation depths for 28 SUs (black bars) and 97 MUs (white bars). C, Distribution of tuning widths for 27 SUs (black bars) and 92 MUs (white bars). Tuning widths were undefined for six units that had maximal evoked rates below the spontaneous rate. D, Distribution of best locations for 23 SUs (black bars) and 75 MUs (white bars). Most units had a contralateral or frontal preference. Best locations were undefined for 27 units that had modulation depths $<50 \%$.

When measured using mean spike rates, most units exhibited only enhancement (firing rates above the spontaneous rate), but an appreciable number of units showed suppressive responses (firing rates below the spontaneous rate). Among our unit population, $39 \%$ of SUs and 25\% of MUs showed a decrease in firing rate more than 2 SEs below the spontaneous rate for at least one source location. Figure 7 illustrates regions of enhancement and regions of suppression in the receptive fields of each of 125 units. Each horizontal row of bars represents a unit. White bars and black bars indicate source locations for which firing rates were significantly increased and decreased, respectively, relative to the spontaneous rate. Black dots indicate unit best locations. Although most units exhibited receptive fields with regions of enhancement only ( $61 \%$ of SUs; $73 \%$ of MUs), we also found units with suppression only ( $18 \%$ of SUs; $11 \%$ of MUs) and units with enhancement at some locations and suppression at others $(21 \%$ of SUs; $13 \%$ of MUs).

\section{Spatial information carried by spike timing}

The analyses presented thus far have dealt with time-averaged spike rates and therefore ignore spike timing. We observed a diversity of temporal response types in our unit population, however, as shown in Figures 4 and 5. If the temporal pattern of the response of a unit varies in a consistent way with sound location, then the pattern should carry information about location. To analyze those temporal patterns, we required a method capable of dealing with multidimensional response measures. The method

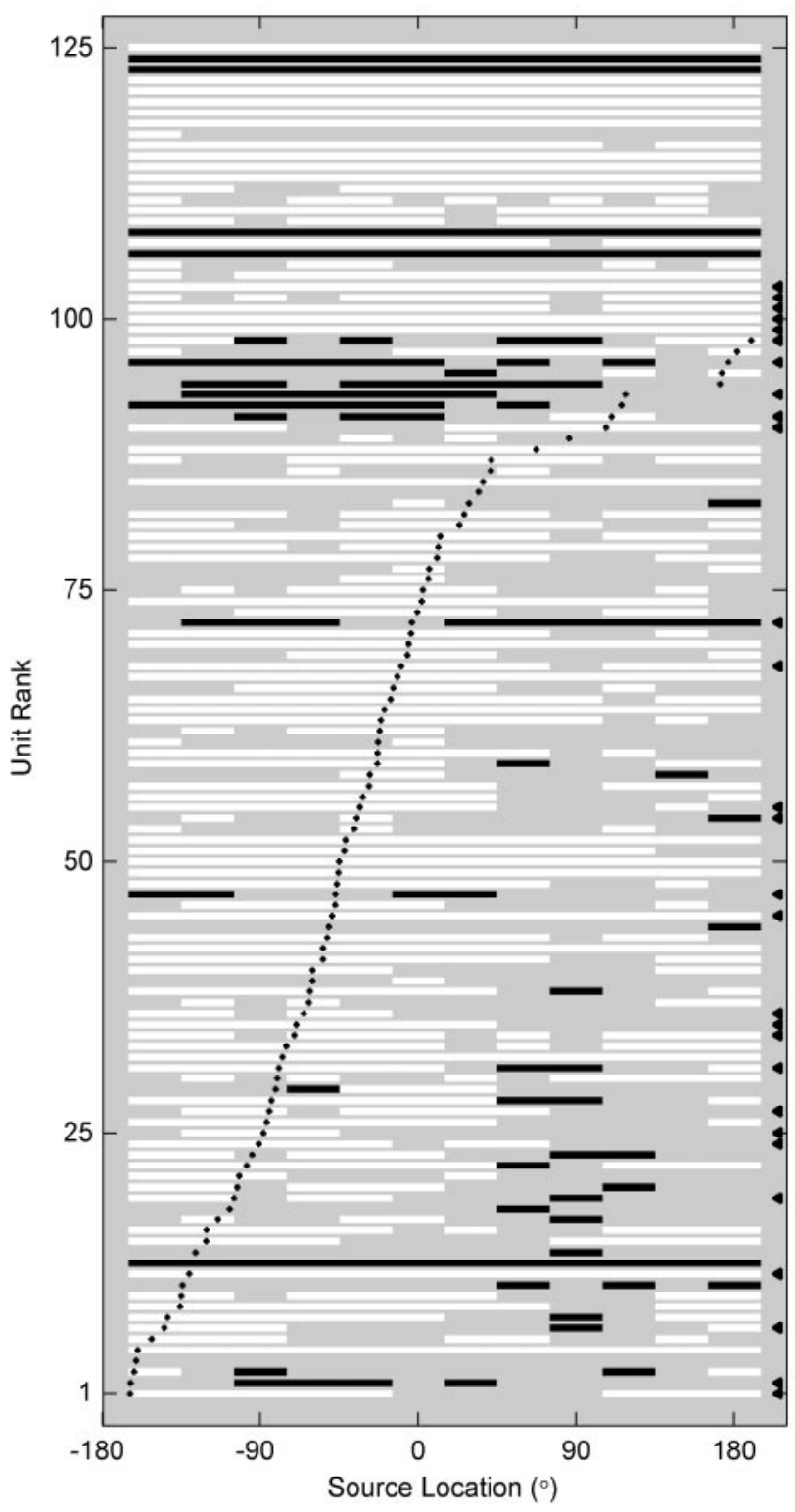

Figure 7. Enhancement and suppression in spatial receptive fields. Each row represents an SU (marked with a black triangle to the right) or an MU. Units are ranked from bottom to top by their best locations, which are indicated by solid black dots. Source location is represented along the abscissa. For each source location, we calculated the mean firing rate and the SEM. White bars signify source locations for which the mean firing rate was $>2$ SEs above the spontaneous rate (enhancement). Black bars indicate a mean firing rate $>2$ SEs below the spontaneous rate (suppression).

that we adopted used a probabilistic artificial neural network to recognize the response pattern of each unit. The network estimated stimulus locations with an accuracy that depended on the discriminability of responses between locations and on the consistency of responses to the same stimulus location. Information theory was used to quantify the accuracy of network estimates. For each unit, we computed the corrected transmitted information, $\hat{T}$, a measure of the amount of location-related information carried by the response of the unit. With 12 stimulus locations, $\hat{T}$ theoretically ranges from 0 to $\sim 3.58$ bits. In practice, noise originating in trial-to-trial variability of unit responses prevented reliable measurement of $\hat{T}$ below $\sim 0.2$ bits.

When this analysis was applied to the full spike patterns of 28 

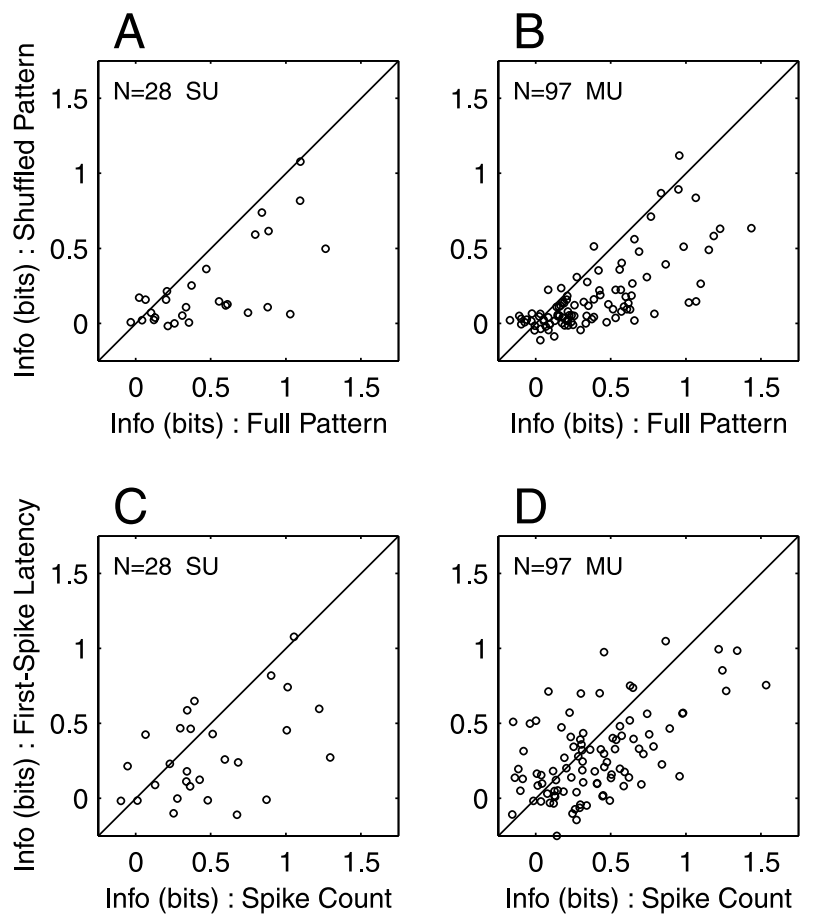

Figure 8. Importance of spike timing in information transmission. $A, B$, Full spike patterns compared with shuffled spike patterns for 28 SUs $(A)$ and 97 MUs $(B)$. Each open circle plots information transmitted about location $(\hat{T})$ for spike patterns in which spike times were randomly shuffled versus $\hat{T}$ for intact spike patterns. The diagonal line indicates equal information under the two conditions. C, D, Spike count compared with first-spike latency for $28 \mathrm{SUs}$ ( $C$ ) and 97 MUs (D). $\hat{T}$ for first-spike latency is plotted against $\hat{T}$ for spike count.

SUs and 97 MUs, $\hat{T}$ ranged from $\sim 0$ to 1.26 bits (mean 0.48 bits, median 0.36 bits) for SUs and $\sim 0$ to 1.44 bits (mean 0.39 bits, median 0.27 bits) for MUs. A detectable amount of information $(>0.2$ bits) was measured in the majority of units (75\% of SUs, $63 \%$ of MUs). Thus the spike patterns of most units transmitted an appreciable amount of location-related information, with the best units transmitting $\sim 40 \%$ of the theoretical maximum.

To estimate the contribution of spike timing to information transmission, we performed the same analysis on shuffled spike patterns, which were created by randomizing the spike times of full patterns. In shuffled patterns, any location-related variation of spike count was preserved, whereas location-related variation of spike timing was eliminated. $\hat{T}$ for shuffled patterns ranged from $\sim 0$ to 1.08 bits (mean 0.24 bits, median 0.12 bits) for SUs and $\sim 0$ to 1.12 bits (mean 0.17 bits, median 0.09 bits) for MUs. The difference in $\hat{T}$ between the full and shuffled conditions, averaged across units, was -0.25 bits for both SUs and MUs. The scatterplots of Figure 8, $A$ and $B$, compare the information transmitted by shuffled patterns with that transmitted by full patterns for each unit in our population. Some units showed nearly equal transmitted information for the two types of spike pattern (symbols near the diagonal), indicating very little information carried by spike timing. The majority of units, however, transmitted less information under the shuffled condition (symbols lying below the diagonal). On average, full patterns transmitted approximately twice as much information as did shuffled patterns. Thus, most units carried a substantial amount of location-related information in spike timing.

Given that spike timing carries information about stimulus location, it is natural to question what precision of spike timing is important. To address the issue, we created interval-shuffled
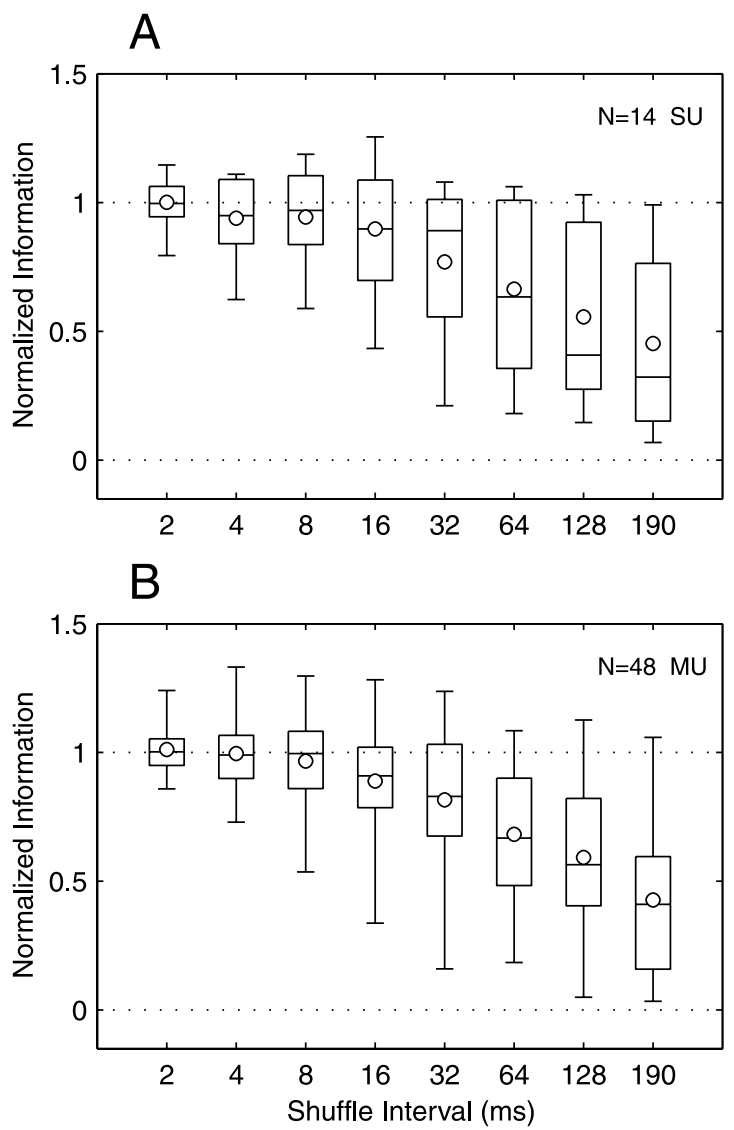

Figure 9. Dependence of information transmission on the precision of spike timing. Transmitted information is plotted versus shuffle interval for 14 SUs $(A)$ and 48 MUs $(B)$. For each unit, full spike patterns were disrupted by randomly shuffling spike times within specified intervals (1-190 msec), and information transmitted about location $(\hat{T})$ was computed for each interval. Information was then expressed relative to $\hat{T}$ for the $1 \mathrm{msec}$ shuffle interval. The best half of the population was selected on the basis of $\hat{T}$ at the $1 \mathrm{msec}$ interval. Circles indicate the mean normalized information across units. Box-and-whisker plots illustrate the distribution of normalized information across units: horizontal lines in a box represent the 25 th, 50 th, and 75 th percentiles, and the lower and higher ends of whiskers represent 5 th and 95 th percentiles.

spike patterns in which spike times were disrupted to an extent determined by the shuffle interval. Spike patterns with larger shuffle intervals had their spike times randomized throughout a greater range of time. For each unit, $\hat{T}$ for each shuffle interval was normalized by dividing by $\hat{T}$ for the $1 \mathrm{msec}$ shuffle interval. The results for the best half of our unit population (as determined by $\hat{T}$ at the 1 msec interval) are represented by box plots in Figure 9. As expected, spike patterns transmitted decreasing amounts of information as the shuffle interval increased; information was minimal at a shuffle interval of $190 \mathrm{msec}$, which corresponded to complete shuffling (as described in Fig. 8A,B). Among this best half of the population, we found that spike patterns preserved their location-related information at shuffle intervals under $16-32 \mathrm{msec}$. At an interval of $32 \mathrm{msec}$, half of the SUs lost $<11 \%$ of the information transmitted with a $1 \mathrm{msec}$ interval. Similarly, at an interval of $16 \mathrm{msec}$, half of the MUs lost $<9 \%$. For both SUs and MUs, the mean normalized information dropped below $90 \%$ between shuffle intervals of 16 and $32 \mathrm{msec}$.

Recent studies in anesthetized animals have suggested that the timing of the first spike after stimulus onset has a dominant role in signaling stimulus location (Brugge et al., 1996; Eggermont, 1998; Furukawa and Middlebrooks, 2002). We addressed this 
question in our preparation by constructing first-spike patterns, which were created from full patterns by discarding all spikes that followed the first spike on each trial. First-spike patterns preserved the mean and dispersion of the latency of the first spike but lacked any variation of spike count or interspike interval with stimulus location. $\hat{T}$ for first-spike patterns ranged from $\sim 0$ to 0.91 bits (mean 0.22 bits, median 0.23 bits) for SUs and $\sim 0$ to 0.92 bits (mean 0.13 bits, median 0.09 bits) for MUs. Thus, less than half of the location-related information present in full spike patterns remained in first-spike patterns. To directly compare information transmission by first-spike latency with that by spike count, we applied to these two unidimensional response measures the same analysis that we applied to spike patterns. On average, $\hat{T}$ for first-spike latency was less than $\hat{T}$ for spike count. $\hat{T}$ for spike count ranged from $\sim 0$ to 1.30 bits (mean 0.50 bits, median 0.38 bits) for SUs and $\sim 0$ to 1.53 bits (mean 0.39 bits, median 0.31 bits) for MUs. $\hat{T}$ for first-spike latency ranged from $\sim 0$ to 1.08 bits (mean 0.29 bits, median 0.24 bits) for SUs and $\sim 0$ to 1.05 bits (mean 0.27 bits, median 0.20 bits) for MUs. The difference in $\hat{T}$ between the spike count and first-spike latency conditions, averaged across units, was -0.21 bits for SUs and -0.11 bits for MUs. The entire unit population is represented in Figure 8, $C$ and $D$. Although some units transmitted more information by first-spike latency (symbols above the diagonal), the majority of units transmitted more information by spike count (symbols below the diagonal). The same analysis was applied to spike dispersion, the SD of spike times. Of the unidimensional response measures, spike dispersion transmitted the least information about stimulus location. $\hat{T}$ for spike dispersion ranged from $\sim 0-0.88$ bits (mean 0.20 bits, median 0.14 bits) for SUs and $\sim 0-0.90$ bits (mean 0.14 bits, median 0.11 bits) for MUs.

As shown in Figures 4 and 5, temporal response patterns were diverse and included onset, sustained, and offset components. We wondered which temporal phases of the response patterns were most important to signaling of stimulus location. To address this question, we estimated $\hat{T}$ as described above except that spike patterns within narrow time windows were used. These windowed spike patterns were $40 \mathrm{msec}$ wide and centered at various peristimulus times. Window centers were $20 \mathrm{msec}$ apart so that neighboring windows overlapped. For each unit, the fractional information was computed for each window by dividing by the sum of $\hat{T}$ across poststimulus time windows and accounting for window overlap. The results from the best half of our unit population (as measured by the sum of $\hat{T}$ across poststimulus time) are shown in Figure 10, $A$ and $B$, respectively. Information about location was transmitted by spikes occurring throughout all temporal phases of the response. Of all time windows, responses centered at $20 \mathrm{msec}$ after stimulus onset transmitted the most information about stimulus location. SUs transmitted on average $56 \%$ of their location-related information within the first 40 msec after stimulus onset; MUs transmitted $44 \%$. For $79 \%$ of SUs and 50\% of MUs, peak information transmission fell within one of the first two time windows (that is, within the first $60 \mathrm{msec}$ after stimulus onset). Nonetheless, later spikes continued to carry location-related information. For instance, for all 14 SUs and for 45 of $48 \mathrm{MUs}$, at least one time window beyond $60 \mathrm{msec}$ contributed $>25 \%$ of the information transmitted.

The diversity of spatially sensitive responses among units suggests that each unit provides information about sound location that is, to some extent, independent of the information transmitted by other units. If this notion is true, then small ensembles of units should transmit appreciably more information about location than do the individual units in the ensemble. We tested this
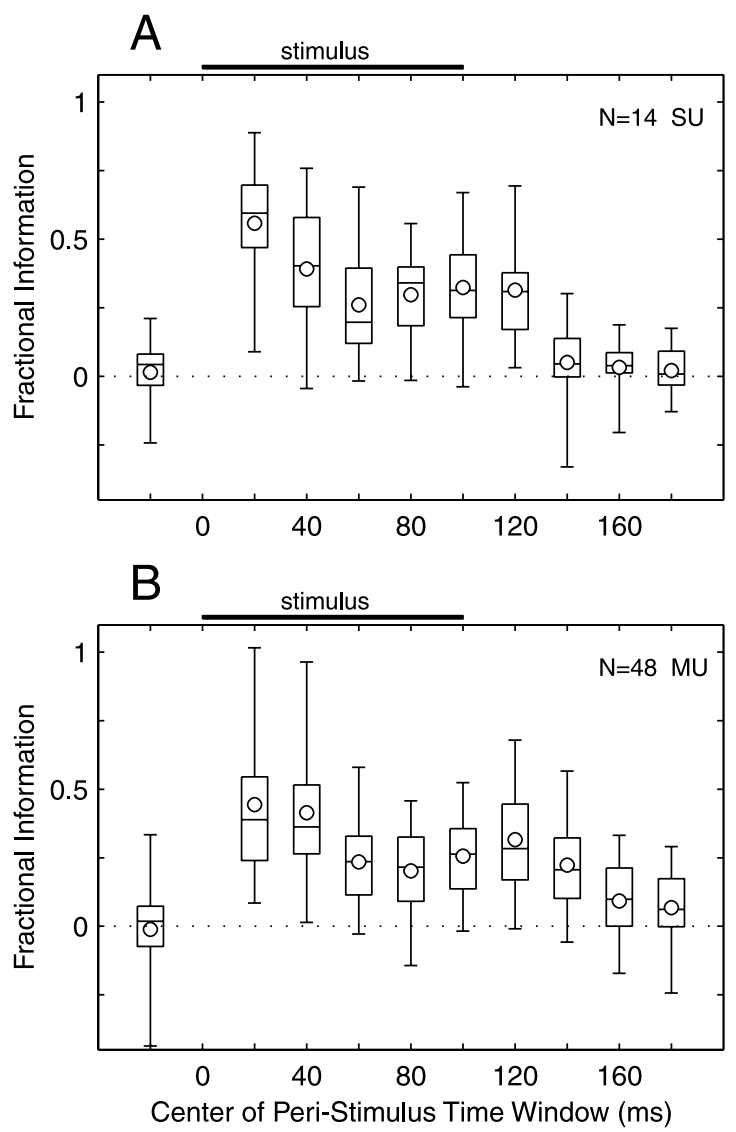

Figure 10. Spikes near stimulus onset transmit the most information. Transmitted information is plotted versus peristimulus time for $14 \mathrm{SUs}(A)$ and $48 \mathrm{MUs}(B)$. For each unit, transmitted information $(\hat{T})$ was computed for windowed spike patterns, which were $40 \mathrm{msec}$ wide and centered at specified peristimulus times. The best half of the population was selected on the basis of the sum of $\hat{T}$ across poststimulus time windows centered from 20 to $180 \mathrm{msec}$. Information within each window was then expressed relative to the sum of $\hat{T}$ across poststimulus time, including a factor that accounts for overlap of adjacent windows. Black bars indicate the time of stimulus presentation. The window centered at -20 msec represents a prestimulus control. Circles indicate the mean fractional information across units. Box-and-whisker plots illustrate the distribution of fractional information across units: horizontal lines in a box represent the 25th, 50th, and 75th percentiles, and the lower and higher ends of whiskers represent 5th and 95th percentiles.

prediction by computing transmitted information $(\hat{T})$ for various ensemble spike patterns, which included the full spike patterns of 2-32 units. Figure 11 shows the results by plotting information as a function of ensemble size. Each symbol represents an ensemble of units. When units were selected randomly for inclusion in ensembles (indicated by crosses), $\hat{T}$ tended to increase monotonically with ensemble size; this trend was clearest when the median was calculated (solid lines). "Best ensembles" (triangles) were constructed from units that individually transmitted the most information; no effort was made to assemble optimal combinations. Best ensembles showed a similar increase in information with ensemble size, but the transmitted information was appreciably higher (up to 2.5 bits). For 11 probe placements, we were able to record from two or more units simultaneously. As shown in Figure 11, those ensembles (circles) transmitted at least as much information as did random ensembles of the same size. This finding indicates that the amount of redundancy in the responses of neighboring units recorded simultaneously was no greater than that in the responses of units sampled randomly from throughout the cortex. 


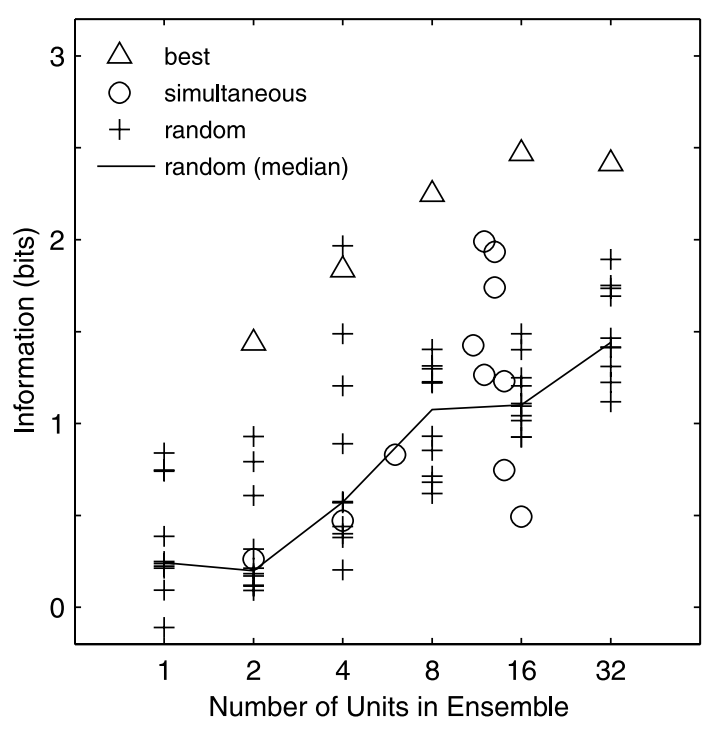

Figure 11. Transmitted information increases with ensemble size. Transmitted information $(\hat{T})$ is plotted for ensemble spike patterns, which included the full spike patterns of 2-32 units (SUs and MUs). Triangles represent ensembles of the best $2-32$ units, as determined by $\hat{T}$ of the individual units. Circles indicate ensembles that were recorded simultaneously on the same recording probe. Crosses represent ensembles consisting of 2-32 units selected randomly without replacement from among 125 units; 10 random ensembles are shown for each ensemble size. For comparison, 10 randomly chosen individual units are also shown. The black curve represents the median for the random condition.

\section{Dependence of spatial tuning on sound level}

The accuracy of localization behavior is known to be relatively independent of sound level for suprathreshold stimuli. In contrast, spatial receptive fields of cortical units in anesthetized cats generally broaden with increasing sound level (Imig et al., 1990; Rajan et al., 1990; Middlebrooks et al., 1998; Stecker et al., 2003). This discrepancy prompted us to investigate the level dependence of spatial tuning in our awake preparation. We recorded 12 SUs and 39 MUs while presenting sounds at three or four sound pressure levels in steps of $10 \mathrm{~dB}$. In contrast to previous studies of anesthetized animals, we found less broadening of spatial tuning with increasing sound level. Four representative units are illustrated in Figure 12. In each panel, a contour plot depicts the mean firing rate of one unit as a function of sound level and sound location. The unit illustrated in Figure $12 \mathrm{~A}$ had a nonmonotonic rate-level function and a relatively level-invariant spatial receptive field: the modulation depth at each level remained $\sim 105 \%$ and the tuning width remained $120-150^{\circ}$ over levels of $30-50 \mathrm{~dB}$ SPL. The unit shown in Figure $12 \mathrm{~B}$ was similarly level invariant but had a monotonic rate-level function. As the sound level increased by $30 \mathrm{~dB}$, the modulation depth decreased only slightly from 88 to $70 \%$, and the tuning width remained $\sim 150^{\circ}$. Another monotonic unit is shown in Figure $12 C$. This unit had a more level-dependent spatial receptive field: with a $20 \mathrm{~dB}$ increase in sound level, the modulation depth remained $\sim 100 \%$, but the tuning width increased from $60^{\circ}$ to $150^{\circ}$. Although this broadening is striking, the receptive field nonetheless remained confined to a hemifield of space. The unit in Figure $12 \mathrm{D}$ was unusual among our population in that its receptive field depended strongly on sound level: as the level increased by $30 \mathrm{~dB}$, the modulation depth decreased from 124 to $41 \%$ and the tuning width increased from $60^{\circ}$ to $270^{\circ}$. Thus, at the highest sound level, this unit responded strongly to sounds throughout three-quarters of auditory space.
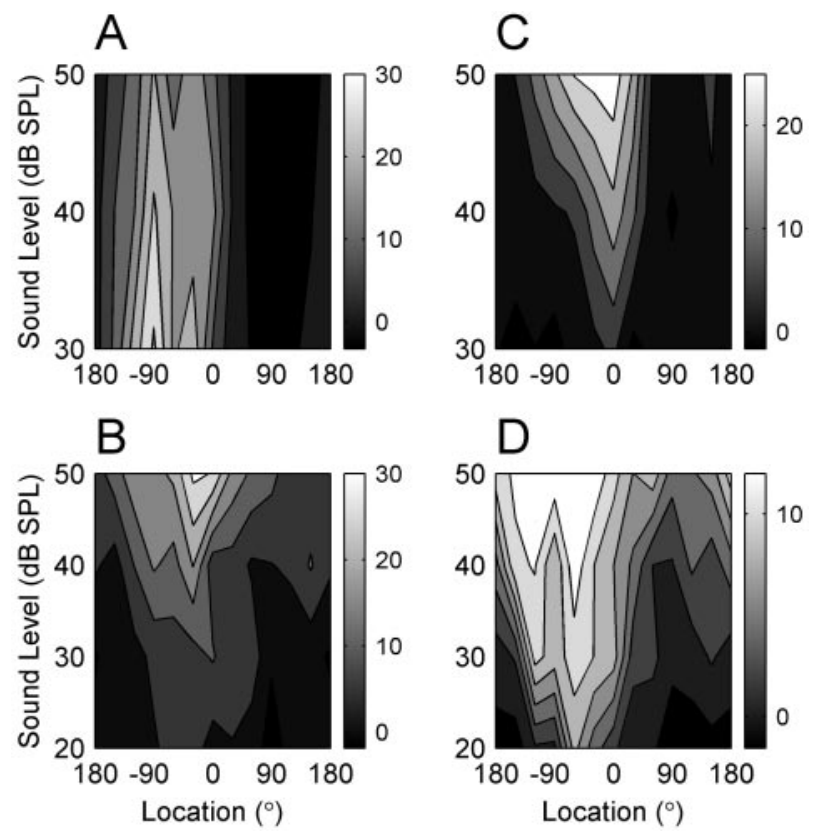

Figure 12. Dependence of spatial receptive fields on sound level. Each panel represents an individual unit. Contour plots show mean spike rate as a function of location and sound level. Vertical bars to the right of each contour plot represent scale bars with spike rate expressed as spikes per second. Two SUs $(A, C)$ and two MUs $(B, D)$ are illustrated.

To characterize the sound-level dependence of receptive fields across our unit population, we analyzed 8 SUs and 23 MUs that were tested at both $30 \mathrm{~dB}$ SPL and $50 \mathrm{~dB}$ SPL. For each unit, tuning statistics (modulation depth, tuning width, and best location) were calculated for each sound level. The change in each statistic was then computed for a change from 30 to $50 \mathrm{~dB}$ SPL. Distributions of the change in each statistic are shown in Figure $13 B-D$. Overall, we found that the modulation depth decreased slightly with increasing level: the mean change was $-10 \%$ for SUs and $-5 \%$ for MUs. Tuning width increased somewhat with increasing level (mean change $\left.23^{\circ} \mathrm{SU}, 20^{\circ} \mathrm{MU}\right)$. Best location also changed to a small extent with sound level (mean change $-14^{\circ} \mathrm{SU},-5^{\circ} \mathrm{MU}$; mean unsigned change $\left.36^{\circ} \mathrm{SU}, 28^{\circ} \mathrm{MU}\right)$. To evaluate the statistical significance of changes in tuning statistics, we estimated $99 \%$ confidence intervals for each tuning statistic and for each unit. The trials at 30 and $50 \mathrm{~dB}$ SPL were separated randomly into two sets (rather than by sound level), and the difference in each tuning statistic between the two sets was calculated. This procedure was repeated 100 times, and the appropriate percentile values were calculated for each tuning statistic. Values falling outside the confidence intervals were considered to contradict the null hypothesis that sound level does not affect spatial receptive fields. By this analysis, 6 of 31 units (19\%) had a significant decrease in modulation depth with increasing sound level; none had a significant increase in modulation depth. No unit was found to have a significant change in tuning width. Best location changed significantly for 4 of 29 units (14\%). For only a small minority of units, therefore, was the null hypothesis rejected.

If spatial receptive fields change only modestly with increasing sound level, one might expect that location signaling would be similarly unperturbed. To test this hypothesis, we computed $\hat{T}$, as described above, from the full spike patterns of 12 SUs and 39 MUs at both 30 and $50 \mathrm{~dB}$ SPL. On average, units transmitted slightly more information at the higher sound level: $\hat{T}$ increased by $18 \%$ for SUs and $13 \%$ for MUs. Many units signaled location more accurately at one level than at the other: of 51 units, 13 

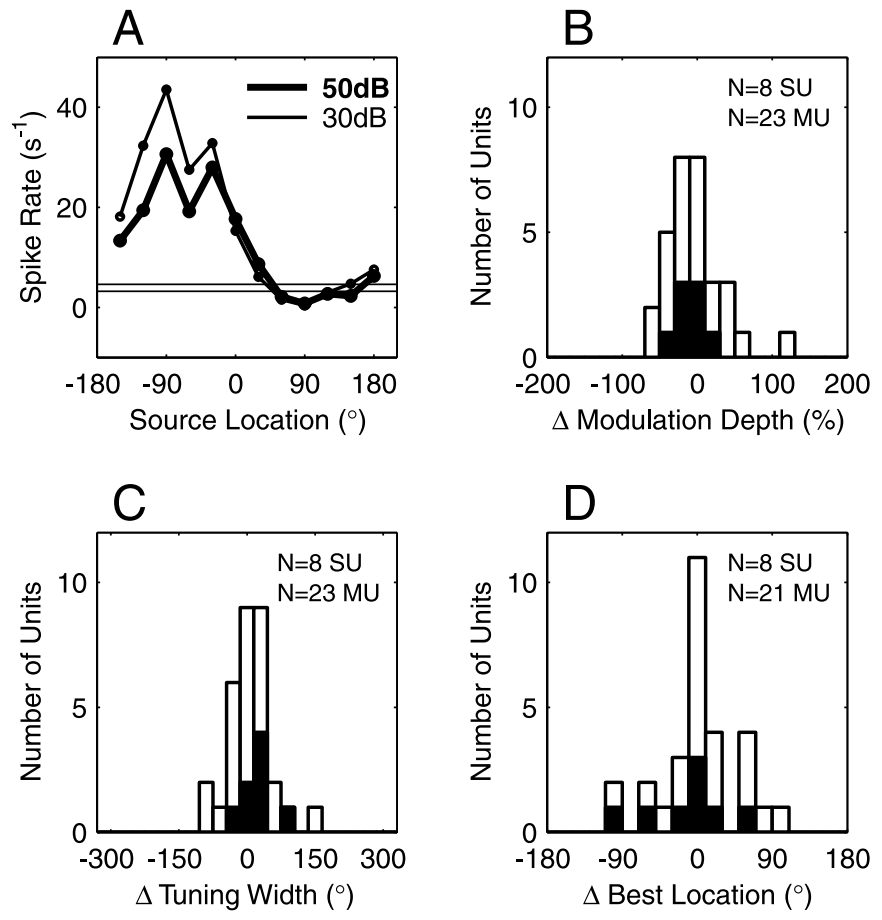

Figure 13. Spatial receptive fields change little with a $20 \mathrm{~dB}$ increase in sound level. $A$, Spatial tuning curve of one SU. The mean firing rate at $30 \mathrm{dBSPL}$ (thin curve, open circles) and at $50 \mathrm{~dB}$ SPL (thick curve, closed circles) is plotted as a function of source location. The thin horizontal lines indicate the spontaneous rate $\pm 1 \mathrm{SE}$. This unit is also shown in Figure $12 A$. $B$, Distribution of change in modulation depth for 8 SUs (black bars) and 23 MUs (white bars). Positive changes indicate a modulation depth that is greater at 50 than at $30 \mathrm{~dB} \mathrm{SPL}$. C, Distribution of change in tuning width for 8 SUs (black bars) and 23 MUs (white bars). D, Distribution of change in best location for 8 SUs (black bars) and 21 MUs (white bars).

(25\%) transmitted $>50 \%$ more information at $30 \mathrm{~dB}$ SPL than at $50 \mathrm{~dB}$ SPL, and $16(31 \%)$ transmitted $>50 \%$ more information at $50 \mathrm{~dB}$ SPL than at $30 \mathrm{~dB}$ SPL. Thus, the accuracy of location signaling was comparable at the two sound levels overall, but some units showed better accuracy at one sound level than at the other. Information transmission remained high under conditions of roving level. When spike patterns at 30 and $50 \mathrm{~dB}$ SPL were pooled together, $\hat{T}$ decreased by only $1 \%$ for SUs and $10 \%$ for MUs, relative to $\hat{T}$ at $30 \mathrm{~dB}$. Thus, spike patterns were similar enough at the two sound levels that our pattern recognition scheme had little trouble generalizing across levels.

\section{Influence of head orientation and behavioral state}

We examined the dependence of unit receptive fields on two potentially important variables that were monitored but not controlled during recording sessions: the absolute orientation of the animal's head and the animal's minute-to-minute behavioral state. We routinely recorded absolute (room-centered) head orientations and then expressed stimulus locations in head-centered coordinates (Fig. 2A). To examine the influence of head orientation, trials were assigned to one of two sets, depending on whether the cat's head was oriented to the left or right of the median head orientation for that recording session. After separation into two sets of trials, 12 SUs and 49 MUs had a sufficient number of trials to allow a comparison. For each unit, tuning statistics were calculated for the head-left and head-right conditions. The difference between the two conditions was then computed for each statistic. Significance of changes in tuning statistics was evaluated by estimating $99 \%$ confidence intervals as de- scribed above in the analysis of responses at different sound levels. Values outside the confidence interval provided evidence to reject the null hypothesis that head orientation does not affect spatial receptive fields. By this analysis, only 3 of 61 units (5\%) had changes in modulation depth associated with head orientation, 1 of 60 units $(1.7 \%)$ had a significant change in tuning width, and 2 of 54 units (4\%) had a change of best location. Thus, few units provided evidence in support of an effect between head orientation and spatial receptive fields.

During recording sessions, the cats' behavioral state alternated between periods during which they were engaged and actively performing the task and periods during which they were idle. We questioned whether changes in the cats' behavioral state might be associated with changes in spatial receptive fields. To examine the question, trials were divided into one of two categories: those during which the cat was performing the behavioral task and those during which the cat was idle. After this categorization, 6 SUs and 28 MUs had a sufficient number of trials in each category to allow a comparison. Tuning statistics were calculated for the performing and idle conditions, and the difference between the two conditions was computed for each statistic. On average, the modulation depth was slightly greater in the performing state relative to the idle state: the difference was $5 \%$ for SUs and $10 \%$ for MUs. Tuning width was decreased in the performing state relative to the idle state: the difference was $5^{\circ}$ for SUs and $23^{\circ}$ for MUs. Best location differed to some extent under the two conditions, but not in a consistent direction (mean absolute change $24^{\circ} \mathrm{SU}, 34^{\circ} \mathrm{MU}$ ). Significance of changes in tuning statistics was determined by $99 \%$ confidence intervals, as described above. Values outside the confidence interval contradicted the null hypothesis that behavioral state has no relation to spatial receptive fields. Only 2 of 34 units (6\%) exhibited a greater modulation depth in the performing state relative to the idle state, and 1 of 34 units (3\%) had a greater modulation depth in the idle state. In 3 of 34 units (9\%), a smaller tuning width was found under the performing condition relative to the idle condition; no units had narrower tuning under the idle condition. Only 2 of 30 units (7\%) had changes in best location. An effect between behavioral state and spatial receptive fields, therefore, was evident for only a small minority of units.

\section{Cortical topography and laminar organization}

A question central to the cortical representation of auditory space is how neurons with various spatially sensitive responses are arranged across cortical areas and across cortical lamina. Our recording technique did not allow us to determine precisely the cortical location or layer of each recording site; however, it did allow simultaneous sampling of unit responses at cortical sites with well defined relative positions. Inspection of plots of unit best location as a function of cortical place from 10 probe placements (4-16 units per probe placement) did not reveal any obvious systematic organization. This was true for seven tangential penetrations (in which recording sites were arranged across the cortical surface), for two radial penetrations (in which sites lay within different cortical lamina), and for one oblique penetration $\left(\sim 45^{\circ}\right.$ angle). For example, the units recorded in the tangential probe placement shown in Figure 5 had best locations (indicated by open triangles) that did not clearly progress with distance across the cortex. Although unit best locations did not show orderly progressions, units with similar best locations were frequently found near each other in the cortex. To analyze this clustering of units, we analyzed pairs of units with known separations $(0-2250 \mu \mathrm{m})$. Linear regression was performed on scatterplots of 
the absolute difference in best location versus site separation for all pairs of units within each probe placement. For 9 of 10 probe placements, the slope of the regression line was positive, suggesting that units that were closer together had more similar best locations, but only 1 penetration reached statistical significance $(p<0.01)$. When all simultaneously recorded pairs of units were taken together $(n=407)$, we found a positive regression slope $\left(21^{\circ} / \mathrm{mm}\right)$ and a weak correlation $\left(r^{2}=0.04\right)$ that was highly significant $(p=0.00006)$. Thus, in our sample we found a weak tendency of neighboring units to have similar best locations, but no orderly map of sound-source location in area A1.

\section{Discussion}

We recorded unit activity from the auditory cortex of awake cats while presenting broadband sounds from loudspeakers positioned throughout $360^{\circ}$ of azimuth. Unit responses were strongly modulated by location, and spatial receptive fields were restricted, in part because of suppression at nonoptimal locations. Receptive fields generally were unchanged over a moderate range of sound level. About half of the location-related information transmitted by units was attributed to variation of spike timing with sound-source location. Spikes after the onset of the sound generally transmitted the most information, but later spikes also carried an appreciable amount of information. Analysis of ensembles of units indicated that the diverse spatial sensitivities of units were, to some degree, mutually independent. We found no evidence of a topographical representation of space in the auditory cortex. Finally, for the vast majority of units, we detected no significant change in spatial receptive fields with the cat's head position or level of participation in the task.

\section{Spatial receptive fields in awake and anesthetized animals}

We are aware of four previous auditory cortical studies that used unanesthetized animals and systematically examined unit responses to sounds at various locations. Three studies investigated unit responses in core and belt areas in awake macaques (Benson et al., 1981; Ahissar et al., 1992; Recanzone et al., 2000), and one described responses in area Al of unanesthetized, paralyzed cats (Eisenman, 1974). Compared with the current study, those studies used stimulus sets that were more limited in the number and range of locations tested. For example, all tested locations were in the frontal hemisphere. Nonetheless, some useful comparisons are possible.

We found that most of the unit responses were strongly modulated by source location: the median modulation depth for SUs in our population (expressed as a percentage of the maximal evoked rate) was $94 \%$. Previous studies have not quantified modulation depth, but examples of units with strongly locationmodulated responses have been illustrated (Benson et al., 1981; Ahissar et al., 1992; Recanzone et al., 2000). We found that $82 \%$ of SUs had modulation depths $>50 \%$ and therefore were considered location sensitive. That figure is consistent with previously reported values (each calculated in a different way), which range from 62 to 92\% (Eisenman, 1974; Benson et al., 1981; Ahissar et al., 1992; Recanzone et al., 2000). It is reasonable that our value falls at the high end of this range, because we tested a greater range of locations. We also found that spatial receptive fields were broad, typically encompassing a hemifield of space, and that the best locations of most units were contralateral or frontal. These findings, too, accord with previous reports (Eisenman, 1974; Benson et al., 1981; Ahissar et al., 1992; Recanzone et al., 2000). One-quarter of our unit population showed suppression of firing rates for at least one source location. It is unclear why previous reports of awake preparations have not explicitly mentioned such suppressive responses, but in at least one case such units may have been screened out before analysis (Benson et al., 1981).

Most of the work on spatial representation in the auditory cortex has used anesthetized animals (for review, see Middlebrooks et al., 2001). General anesthetics are known to affect the spontaneous rate, the temporal dynamics of responses, the magnitude of responses, and the binaural response properties of auditory cortical neurons, although it is clear that not all anesthetic protocols are equivalent (Pfingst et al., 1977; Zurita et al., 1994; Fitzpatrick et al., 2000; Cheung et al., 2001). Despite the known effects, the influence of anesthetic agents on spatial receptive fields in particular has not been well described. In the current work, we did not directly compare unit responses with and without general anesthesia. Nonetheless we are able to compare our findings with the results obtained previously in our laboratory under similar stimulus conditions but with chloralose anesthesia (Middlebrooks et al., 1998; Stecker et al., 2003). In the awake preparation, the median modulation depth for area A1 was $94 \%$ for SUs and 77\% for MUs. In anesthetized cats, the median modulation depth was $55-75 \%$ for MUs in area A1 and $44-86 \%$ for units in areas posterior auditory field (PAF), A2, and anterior ectosylvian sulcus auditory area (AES). Modulation depths were thus slightly higher in the absence of anesthesia. We found that the median tuning width was $150^{\circ}$ for SUs and $180^{\circ}$ for MUs under awake conditions. In contrast, median values in anesthetized animals were $270-350^{\circ}$ for MUs in area A1 and $240-360^{\circ}$ for units in areas PAF, A2, and AES. Thus spatial tuning was markedly narrower in awake animals.

To examine the dependence of spatial receptive fields on sound level, we presented stimuli over a range of moderate levels. We found that the modulation depths, tuning widths, and best locations of units were essentially preserved over a range of $10-40 \mathrm{~dB}$ above unit threshold. In particular, tuning widths increased by only $20-30^{\circ}$ with an increase in sound level of $20 \mathrm{~dB}$. Previous studies have not systematically examined the influence of sound level in the absence of anesthesia, but a qualitative study by Eisenman using paralyzed cats showed that "in most instances" receptive fields were similar across a range of 20 or $30 \mathrm{~dB}$ (Eisenman, 1974). This level invariance contrasts with the broadening of receptive fields seen in anesthetized preparations as the sound level is increased. For instance, a $20 \mathrm{~dB}$ increase in sound level increased the median tuning width of MUs in area A1 by $\sim 80^{\circ}$ (Stecker et al., 2003). The narrower tuning and resistance to broadening at higher levels that is found in the awake state is likely attributable in part to suppression below the spontaneous rate at nonoptimal stimulus locations. This mechanism may act to improve stimulus selectivity more generally in sensory cortex, because a similar finding has been reported in macaque V1 (Ringach et al., 2002).

\section{Spatial coding by spike timing}

Recent work using anesthetized animals has suggested that spike timing is important in the representation of sound location. For example, SUs in area A2 transmitted only $65 \%$ as much information when location-related variation in spike timing was removed before computing transmitted information (Furukawa and Middlebrooks, 2002). In agreement with this result, we found that SUs in area A1 of awake cats transmitted only $50 \%$ as much information when timing was obliterated. Studies of A1 and A2 in anesthetized animals have shown that, in particular, the timing of the first spike carries much of the information about location (Brugge et al., 1996; Eggermont, 1998; Furukawa and Middle- 
brooks, 2002). In one study, first-spike latency was found to carry more information than spike count (Furukawa and Middlebrooks, 2002). In contrast, we found in awake cats that first-spike latency carries less information about source location than does mean firing rate (i.e., spike count). This finding may be related to the fact that, under anesthesia, units typically respond to a stimulus with one or a few spikes. Although a single spike in principle can provide a continuous range of spike times as part of a latency code, it provides only limited possibilities for a rate code. In contrast, in the absence of anesthesia, units respond with a greater number of spikes, which allows for a greater range of stimulus-modulated spike rates. Another reason that first-spike latency codes location less effectively in the absence of anesthesia may be that the first spike can be obscured by background spontaneous activity.

\section{Movements of the head, pinnae, and eyes}

Unlike most previous studies, in which the animal's head was fixed in place, the cats in our experiments were free to move their heads. This feature allowed us to coarsely analyze the dependence of spatial receptive fields on the orientation of the head. Our approach was to assume that receptive fields were head centered and to look for receptive fields that deviated from this assumption. We found that $<5 \%$ of units showed significant changes in spatial receptive fields, suggesting that the head-centered reference frame is indeed the most appropriate. To our knowledge, the only other study to address this question is a qualitative study of the temporoparietal cortex of macaques that included a number of unimodal auditory units in belt and parabelt cortex (Leinonen et al., 1980). Those investigators reported that for the few cells that were examined, "responses depended on the angle of incidence of the sound with reference to the head rather than to the body." Although we tracked the orientation of the head, we did not track the pinnae and eyes. A recent study of the macaque inferior colliculus suggested that unit responses depend on the direction of gaze (Groh et al., 2001). Further studies would be required to determine whether such an effect is present in the auditory cortex. We would expect a dependence on eye position in our experiments to increase the variability of responses and broaden apparent receptive fields, neither of which can be ruled out. Passive displacements of the pinnae in anesthetized animals have been shown to alter spatial receptive fields in the dorsal cochlear nucleus, the inferior colliculus, the superior colliculus, and the auditory cortex (Middlebrooks and Pettigrew, 1981; Aitkin et al., 1984; Middlebrooks and Knudsen, 1987; Imig et al., 2000). We believe that the slight and infrequent pinna movements observed under our experimental conditions likely had only small effects on unit responses.

\section{Conclusion}

In summary, we found that neurons in the primary auditory cortex of awake cats represented the locations of sounds with diverse temporal responses. Future studies of other cortical fields and locations well off the horizontal plane are warranted. We expect that future investigations will also describe ways in which responses are influenced by the animal's behavior (especially sound localization behavior) and by immediately preceding stimuli, i.e., by the sensory context.

\section{References}

Ahissar M, Ahissar E, Bergman H, Vaadia E (1992) Encoding of soundsource location and movement: activity of single neurons and interactions between adjacent neurons in the monkey auditory cortex. J Neurophysiol 67:203-215.

Aitkin LM, Gates GR, Phillips SC (1984) Responses of neurons in inferior colliculus to variations in sound-source azimuth. J Neurophysiol 52:1-17.

Anderson DJ, Najafi K, Tanghe SJ, Evans DA, Levy KL, Hetke JF, Xue X, Zappia JJ, Wise KD (1989) Batch-fabricated thin-film electrodes for stimulation of the central auditory system. IEEE Trans Biomed Eng 36:693-704.

Benson DA, Hienz RD, Goldstein MH (1981) Single-unit activity in the auditory cortex of monkeys actively localizing sound sources: spatial tuning and behavioral dependency. Brain Res 219:249-267.

Bierer SM, Anderson DJ (1999) Multi-channel spike detection and sorting using an array processing technique. Neurocomputing 26-27:947-956.

Brugge JF, Reale RA, Hind JE (1996) The structure of spatial receptive fields of neurons in primary auditory cortex of the cat. J Neurosci 16:4420-4437.

Cheung SW, Nagarajan SS, Bedenbaugh PH, Schreiner CE, Wang X, Wong A (2001) Auditory cortical neuron response differences under isoflurane versus pentobarbital anesthesia. Hear Res 156:115-127.

Demuth H, Beale M (2000) Neural network toolbox user's guide, Version 4. Natick, MA: MathWorks.

Eggermont JJ (1998) Azimuth coding in primary auditory cortex of the cat. II. Relative latency and interspike interval representation. J Neurophysiol 80:2151-2161.

Eisenman LM (1974) Neural encoding of sound location: an electrophysiological study in auditory cortex (AI) of the cat using free field stimuli. Brain Res 75:203-214.

Fitzpatrick DC, Kuwada S, Batra R (2000) Neural sensitivity to interaural time differences: beyond the Jeffress model. J Neurosci 20:1605-1615.

Furukawa S, Middlebrooks JC (2001) Sensitivity of auditory cortical neurons to locations of signals and competing noise sources. J Neurophysiol 86:226-240.

Furukawa S, Middlebrooks JC (2002) Cortical representation of auditory space: information-bearing features of spike patterns. J Neurophysiol 87:1749-1762.

Furukawa S, Xu L, Middlebrooks JC (2000) Coding of sound-source location by ensembles of cortical neurons. J Neurosci 20:1216-1228.

Good MD, Gilkey RH (1996) Sound localization in noise: the effect of signal-to-noise ratio. J Acoust Soc Am 99:1108-1117.

Groh JM, Trause AS, Underhill AM, Clark KR, Inati S (2001) Eye position influences auditory responses in primate inferior colliculus. Neuron 29:509-518.

Heffner HE (1997) The role of macaque auditory cortex in sound localization. Acta Otolaryngol 532:22-27.

Heffner HE, Heffner RS (1990) Effect of bilateral auditory cortex lesions on sound localization in Japanese macaques. J Neurophysiol 64:915-931.

Imig TJ, Irons WA, Samson FR (1990) Single-unit selectivity to azimuthal direction and sound pressure level of noise bursts in cat high-frequency primary auditory cortex. J Neurophysiol 63:1448-1466.

Imig TJ, Bibikov NG, Poirier P, Samson FR (2000) Directionality derived from pinna-cue spectral notches in cat dorsal cochlear nucleus. J Neurophysiol 83:907-925.

Jenison RL, Schnupp JW, Reale RA, Brugge JF (2001) Auditory space-time receptive field dynamics revealed by spherical white-noise analysis. J Neurosci 21:4408-4415.

Jenkins WM, Masterton RB (1982) Sound localization: effects of unilateral lesions in central auditory system. J Neurophysiol 47:987-1016.

Jenkins WM, Merzenich MM (1984) Role of cat primary auditory cortex for sound-localization behavior. J Neurophysiol 52:819-847.

Kavanagh GL, Kelly JB (1987) Contribution of auditory cortex to sound localization by the ferret (Mustela putorius). J Neurophysiol 57:1746-1766.

Leinonen L, Hyvarinen J, Sovijarvi AR (1980) Functional properties of neurons in the temporo-parietal association cortex of awake monkey. Exp Brain Res 39:203-215.

Macpherson EA, Middlebrooks JC (2000) Localization of brief sounds: effects of level and background noise. J Acoust Soc Am 108:1834-1849.

Mickey BJ, Middlebrooks JC (2001) Responses of auditory cortical neurons to pairs of sounds: correlates of fusion and localization. J Neurophysiol 86:1333-1350.

Middlebrooks JC (1992) Narrow-band sound localization related to external ear acoustics. J Acoust Soc Am 92:2607-2624. 
Middlebrooks JC, Knudsen EI (1987) Changes in external ear position modify the spatial tuning of auditory units in the cat's superior colliculus. J Neurophysiol 57:672-687.

Middlebrooks JC, Pettigrew JD (1981) Functional classes of neurons in primary auditory cortex of the cat distinguished by sensitivity to sound location. J Neurosci 1:107-120.

Middlebrooks JC, Clock AE, Xu L, Green DM (1994) A panoramic code for sound location by cortical neurons. Science 264:842-844.

Middlebrooks JC, Xu L, Eddins AC, Green DM (1998) Codes for soundsource location in nontonotopic auditory cortex. J Neurophysiol 80:863-881.

Middlebrooks JC, Xu L, Furukawa S, Mickey BJ (2001) Location signaling by cortical neurons. In: Integrative functions in the mammalian auditory pathway (Oertel D, Popper AN, Fay RR, eds), pp 319-357. New York: Springer.

Morimoto M, Hitoshi A (1984) Localization cues of sound sources in the upper hemisphere. J Acoust Soc Jpn 5:165-173.

Pfingst BE, O'Connor TA, Miller JM (1977) Response plasticity of neurons in auditory cortex of the rhesus monkey. Exp Brain Res 29:393-404.

Populin LC, Yin TC (1998) Pinna movements of the cat during sound localization. J Neurosci 18:4233-4243.

Rajan R, Aitkin LM, Irvine DR, McKay J (1990) Azimuthal sensitivity of neurons in primary auditory cortex of cats. I. Types of sensitivity and the effects of variations in stimulus parameters. J Neurophysiol 64:872-887.

Reale RA, Imig TJ (1980) Tonotopic organization in auditory cortex of the cat. J Comp Neurol 192:265-291.

Reale RA, Jenison RL, Brugge JF (2003) Directional sensitivity of neurons in the primary auditory (AI) cortex: effects of sound-source intensity level. J Neurophysiol 89:1024-1038.

Recanzone GH, Guard DC, Phan ML, Su TK (2000) Correlation between the activity of single auditory cortical neurons and sound-localization behavior in the macaque monkey. J Neurophysiol 83:2723-2739.

Rieke F, Warland D, de Ruyter van Steveninck R, Bialek W (1997) Spikes. Cambridge, MA: MIT.

Ringach DL, Bredfeldt CE, Shapley RM, Hawken MJ (2002) Suppression of neural responses to non-optimal stimuli correlates with tuning selectivity in macaque V1. J Neurophysiol 87:1018-1027.

Stecker GC, Mickey BJ, Macpherson EA, Middlebrooks JC (2003) Spatial sensitivity in field PAF of cat auditory cortex. J Neurophysiol 89:2889-2903.

Su TI, Recanzone GH (2001) Differential effect of near-threshold stimulus intensities on sound localization performance in azimuth and elevation in normal human subjects. J Assoc Res Otolaryngol 2:246-256.

Thompson GC, Cortez AM (1983) The inability of squirrel monkeys to localize sound after unilateral ablation of auditory cortex. Behav Brain Res 8:211-216.

Xu L, Furukawa S, Middlebrooks JC (1998) Sensitivity to sound-source elevation in nontonotopic auditory cortex. J Neurophysiol 80:882-894.

Zhou B, Green DM, Middlebrooks JC (1992) Characterization of external ear impulse responses using Golay codes. J Acoust Soc Am 92:1169-1171.

Zurita P, Villa AE, de Ribaupierre Y, de Ribaupierre F, Rouiller EM (1994) Changes of single unit activity in the cat's auditory thalamus and cortex associated to different anesthetic conditions. Neurosci Res 19:303-316. 